Int. J. Dev. Biol. 57: 415-425 (2013)

doi: $10.1387 /$ ijdb.120256hk

\title{
Unc-5/netrin-mediated axonal projection during larval serotonergic nervous system formation in the sea urchin, Hemicentrotus pulcherrimus
}

\author{
KOUKI ABE, TOMOKO KATOW, SHIOH OOKA and HIDEKI KATOW* \\ Research Center for Marine Biology, Tohoku University, Asamushi, Aomori, Japan
}

\begin{abstract}
The molecular structure and role of two splice-isoforms of Unc-5 (Hp-Unc-5v1 and v2) in Unc-5/netrin interaction during serotonergic axonal projection were elucidated in this study. HpUnc-5v1 was found to be comprised of two immunoglobulin-like domains, two thrombospondin domains in the extracellular region, and ZU-5, DB, and Death domains in the cytoplasmic region, whereas Hp-Unc-5v2 lacked one thrombospondin domain, the transmembrane domain, and all cytoplasmic domains. Hp-Unc-5v1 was transcribed in unfertilized eggs, which continued until the 3-day post-fertilization (-dpf) 2-arm pluteus stage, but was suspended at the mesenchyme blastula stage ( $\mathrm{mBI}$ ), whereas Hp-Unc-5v2 was not transcribed in unfertilized eggs, but was from after fertilization to the same developmental stage of $\mathrm{mBI}$ as Hp-Unc-5v1. Relative accumulation of transcripts of both splice-isoforms peaked at the prism stage and declined thereafter, and they were localized at the vegetal pole region of early gastrulae, around the blastopore in mid- to late gastrulae, at fore- and mid-gut regions and on the basal side of dorsal ectoderm in 28-hour postfertilization prism larvae, and within axons at and after the 2-dpf pluteus stage. Hp-Unc-5v2:GFP was detected in the entire serotonergic cell body and extracellularly on the basal surface of oral ectoderm in 2-dpf plutei and exclusively within axons in 4-dpf plutei. Overexpression of Hp-Unc-5v2 resulted in decreased axonal projection in plutei. Knockdown of Hp-unc-5v1 and v2 by morpholino antisense oligonucleotide resulted in severe deficiency of axonal projection. Interference of Unc-5/ netrin interaction using an exogenous synthetic IFKSODFGKWOPY peptide from the VI domain in Hp-netrin, inhibited axonal projection and larval swimming.
\end{abstract}

KEY WORDS: axonal projection, Unc-5 variant, netrin, serotonergic axon, sea urchin larvae

\section{Introduction}

The serotonergic nervous system (SNS) of sea urchin larvae plays a major role (Yaguchi and Katow, 2003; Katow et al., 2007) along with the dopaminergic regulatory system (Katow et al., 2010) and the GABAergic nervous system (Katow et al., 2013) in swimming activity. SNS is the last system to emerge among the above three classic nervous systems during the prism larva stage, which is initiated by ganglion formation at the apical ectoderm of the oral lobe (Yaguchi et al., 2000; Katow et al., 2013). The projection of serotonergic axons occurs under the regulation of netrin on the basal surface of oral ectoderm at the larval apical ganglion during the early pluteus stage. Initial axonal projection occurs toward the netrin-rich dorsal middle-belt that is formed along the antero-posterior axis of larva, and concomitantly during crossing the middle-belt, away from the netrin-rich area in the contralateral direction (Katow 2008).

Such contradictory responses of axons to netrin have been widely acknowledged in commissural axons as they cross the midline in the central nervous system of vertebrates and invertebrates (Van Vactor and Lorenz, 1999; Araújo and Tear, 2003); they are mediated by attraction via Deleted in Colorectal Cancer (DCC) family

Abbreviations used in this paper:-dpf, day post-fertilization; -hpf, hour post-fertilization; SNS, serotonergic nervous system; V1, Hp-Unc-5 variant 1; V2, Hp-Unc-5 variant 2 .

\footnotetext{
*Address correspondence to: Hideki Katow. Research Center for Marine Biology, Tohoku University, Asamushi, Aomori, Aomori 039-3501, Japan.

E-mail: hkatow@m.tohoku.ac.jp
}

Supplementary Material (four figures) for this paper is available at: http://dx.doi.org/10.138710.1387/ijdb.120256hk 
receptors and repulsion via Unc-5 family receptors (Barallobre et al., 2005; Sun et al., 2011; Norris and Lundquist, 2011).

In this study, we report (1) the identification and structural analysis of two splice-isoforms of Unc-5 receptors of larvae of the sea

1 GAAAATTCATTGGTAGCATTGGAGCTAGATGCCTCACGCATGCTGCGATTGGCTGTTATCCAATACCACATTCTCATCTGCATGGAATCT 90 91 TAACAAGTTCAGGGACTTCGCATCTAATATCTCTTTTTCAAGGCAGTCTTGATGGAGACCCGAGTGGCTTAGGAGCGAGTCAGGAGCCGC 180 181 CGAAAAGTCATCGTGATTATTACTTGGTGGAGCTACTACCCCGAAAGGATACTGGAGATCTATACCCACATCACCGGCAAGATGAGACGC 270 1 M R R 3

271 CGAAGTGCCGGGCTAAGGATTCATGAGGTTACCGTTGTGCTTGTGCTGTTTGGTGGGATTGCATCGAGGCCAGTTTTAGGACAACTTGAT 360

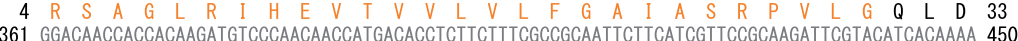
$\begin{array}{llllllllllllllllllllllllllllllll}34 & G & Q & P & P & Q & D & V & P & T & T & M & T & P & L & L & S & P & Q & F & F & I & V & P & Q & D & S & Y & I & T & K & 63\end{array}$ 451 AGAAGCGTGGATCTAGAATGCAAGGCCGGTCCTTCTCCCAATGTGTACTTTATATGCAATGATGAGAAGATAGCTGATGCCAGGACACAT 540 $64 \mathrm{R} S \mathrm{~S}$ V D L E C K A G P S P N V Y F I C N D E K I A D A R T H 93 541 TCTCGTGGTACCTTTGATGAATACTATGAGAATATCAGACATATAGCTTTAACCATAACAAAAGATGAGGTGCAAGAGTACTTTGGTGAT 630 $\begin{array}{lllllllllllllllllllllllllllllllll}94 & S & R & G & T & F & D & E & Y & Y & E & N & I & R & H & I & A & L & T & I & T & K & D & E & V & Q & E & Y & F & G & D & 123\end{array}$ 631 GAGGATTTCTGGTGCGTCTGCGAAGCCGCTGCTGATCCACAGCCAATCAGGACAGAGAAGGCCTATATCAGAGAAGCATACCTGCGTAAA 720 124 E 721 CAGTTCCTCCAGATGCCACTGGACCACAGTGTGCCCCTCCATGACAAGTTCCATCTCCTGTGCCGGGCACCAGAGGGCGTCCCAGAGCCG 810 $\begin{array}{lllllllllllllllllllllllllllllllll}154 & Q & F & L & Q & M & P & L & D & H & S & V & P & L & H & D & K & F & H & L & L & C & R & A & P & E & G & V & P & E & P & 183\end{array}$ 811 ACCATCCATTGGGAGATTGACGGAGTGCCCATTGACGACGAGAACCTCGTGCACTACGTGGTAACATACGACGGGACCCTCATCGTCAAT 900 184 T I $\quad H \quad W \quad E$ I $D$ G 901 GAAGCTACACTGGCAGATAATGGCAACTATACATGTGTCGCCACCAATGTTGCCACTTACAGAACAACAGATCCAGCTCGTGTCATTGTA 990 $\begin{array}{llllllllllllllllllllllllllllllll}214 & E & A & T & L & A & D & N & G & N & Y & T & C & V & A & T & N & V & A & T & Y & R & T & T & D & P & A & R & V & I & V & 243\end{array}$ 991 TACGATAATACAAACGATGGAGCATGGACGATGTGGACAGAGTGGAGCACCTGTACCGGAGACTGTGACGGTGGGACCAGGAGAAGgATG 1080 244 Y D N N T N D G A W T M W T E W S T C T G D C D G G T R R R M 273 1081 CGATACTGCACCAACCCTGCCCCTCTATCGATGGAGCCAACTGCGCGGGAAAGGCATTGCAGACCGAAGACTGTTCAATCGATTGCCCAG 1170 274 R Y $\quad$ C T T N P A P L S M E P T A R E R H C R P K T V Q S I A 1171 GTGCGGCCATTGGTTGGAGCGAATGGAGCGTGTGGTCCCGCATGCACGGACGAGTGTGAACAGATCAGGACCAGGACCTGCACCAGGCAA 1260 $\begin{array}{lllllllllllllllllllllllllllllllll}304 & V & R & P & L & V & G & A & N & G & A & C & G & P & A & C & T & D & E & C & E & Q & I & R & T & R & T & C & T & R & Q & 333\end{array}$

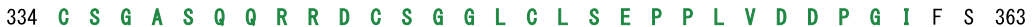
1351 CCTAGTGCTGTAGACACAAGGTCTCAAAACCCAGCTGCTGGCAGCAAGAATGGTCTCTCCAAGCAGATCCCAGTTTACATCGGCATCTCT 1440 $\begin{array}{llllllllllllllllllllllllllllllll}364 & P & S & A & V & D & T & T & S & Q & N & P & A & A & G & S & K & N & G & L & S & K & Q & I & P & V & Y & I & G & I & S & 393\end{array}$ 1441 CTAGCCATTGTCGTGCTCCTTCTCGTCTTCCTCTTCATCGCTATCTACCTAGTCACCAAGAGAAAAAGGGGAAACTCCCCCAGCTATACC 1530 $\begin{array}{llllllllllllllllllllllllllllllll}394 & \mathrm{~L} & A & \text { I } & V & V & \mathrm{~L} & \mathrm{~L} & \mathrm{~L} & V & \mathrm{~F} & \mathrm{~L} & \mathrm{~F} & \text { I } & A & \text { I } & Y & \mathrm{~L} & V & \mathrm{~T} & \mathrm{~K} & \mathrm{R} & \mathrm{K} & \mathrm{R} & \mathrm{G} & \mathrm{N} & \mathrm{S} & \mathrm{P} & \mathrm{S} & \mathrm{Y} & \mathrm{T} & 423\end{array}$ 1531 ACCACCTCCACCGAGGATTGTAACCTGGCTATGCTTTCAGCACAACCACCAGACATCACTACCCAGACGATGCACTCCTCGCTACGATCC 1620 $\begin{array}{lllllllllllllllllllllllllllllllll}424 & T & T & S & T & E & D & C & N & L & A & M & L & S & A & Q & P & P & D & I & T & T & Q & T & M & H & S & S & L & R & S & 453\end{array}$ 621 AACCACGTCGCCCTCTCAAGTCACAACGAGAAGATTCCGATGTCTGGGACACCGACGCATCCACCCCATCCTGACCTGACGGGCGGCAAG 1710

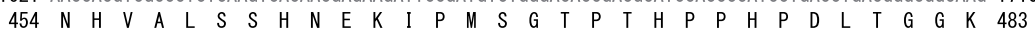
1711 ATCCCACATCAGCTTACCGTTCAGTTCTCGCCCAAAAAAGGAGGGACCATAACGCAGCTTGGGTGCTTGGGGTCGTCGGATATCTCACAG 1800

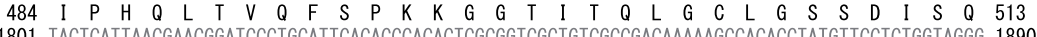
801 TACTCATTAACGAACGGATCCCTGCATTCACACCCACACTCGCGGTCGCTGTCGCCGACAAAAAGCCACACCTATGTTCCTCTGGTAGGG 1890 $\begin{array}{llllllllllllllllllllllllllllllll}514 & Y & S & L & T & N & G & S & L & H & S & H & P & H & S & R & S & L & S & P & T & K & S & H & T & Y & V & P & L & V & G & 543\end{array}$ 891 CCTGGACAGGAGAAACACATCTACGCCACACTGAATCCTCCAGAACCACCTCCACAGGATCCCAGATACTGTCAGACCCGTTGCTACAGC 1980 $544 \mathrm{P} G Q \mathrm{G}$ E K H I Y A T L N P P E P P P Q D P R Y C Q T R C Y S 573 1981 GACTTGGAGgACAATGATGgGTACATTATGgAGGAGgCGgATGAAAACTACATGCTGCAGgGACATTCCTCAGAGGAAAGTCTTGAGCCA 2070

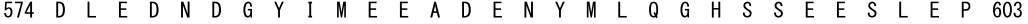
2071 CAGTCTATGTGTGATTCCGATCCAGGCAGGACATTATCAGGCAGTGCTCTAGGGCTTCCAATGCATTCGGATTACAGCATAGCAACGGGA 2160 $\begin{array}{llllllllllllllllllllllllllllllll}604 & Q & S & M & C & D & S & D & P & G & R & T & L & S & G & S & A & L & G & L & P & M & H & S & D & Y & S & I & A & T & G & 633\end{array}$ 2161 CATGTTGGATCAAGAGGGGGCAGATTAGTGCTACCAGATTCAGGAGTTAGCTTGATGGTACCTGAGGGTGCCATAGCACGCGGCCAGACG 2250 634 H V G S R G G R L V L P D S G V S L M V P E G A I A R G O T 663 2251 GTGGAAATATACCTAGCAGTCTCACATGAACTAATGGACAGACCGCACATAGAGCACAACCAGACCTTACTTAGCCCCGTCATCTTGTGC 2340 664 V E I Y L A V S H E L M D R P H I E H N Q T L L S P V I L C 693 2341 GGCCCGCCCTCTGTAGTTCTTGCCAAGTCAGTCGTCTTGGTGTTACCGCACTGCGCACAGATGAGCAAGAACGACTGGAGGCATTCGGTC 2430 694 G P P S V V L A K S V V L V L P H C A Q M S K N D W R H S V 723 2431 ATCGCCAGCAGTACACATCCAACCGATGATAAATGTTGGGAGAAGCAGACAACAGTCGGAGAGGAAACCATCAACTTACAAGTCTTCTGC 2520

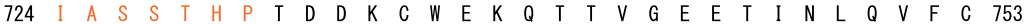
2521 CAGGTGGATAGTCATCAATCCTGCATCGTGACCGATCAGCTCGGGTGGTACGCCCTCAGTGGCGAGTCAAGGCCAGAGAGACAGTCGGGG 2610 $\begin{array}{llllllllllllllllllllllllllllllll}754 & Q & V & D & S & H & Q & S & C & I & V & T & D & Q & L & G & W & Y & A & L & S & G & E & S & R & P & E & R & Q & S & A & 783\end{array}$ 2611 AAGAGGTTAAAACTGTTAGCATTCGGTCCAGCTCTTCGGTCAAGGCTAGATTACCATATCAAAGTGTACATCTCGGAAGACACTCCAGAT 2700 $\begin{array}{lllllllllllllllllllllllllllllllll}784 & K & R & L & K & L & L & A & F & G & P & A & L & R & S & T & L & D & Y & H & I & K & V & Y & I & S & E & D & T & P & D & 813\end{array}$ 2701 GCTATAGAGCACATCCTCAATGTTGAGAGGAGACTCGGAGGACAGCCCCTCCAGGGTAGCTGCCAATTTGACTACCACGACAATGGCAAT 2790

814 A I 2791 AATCTCAGATTGGAGATCCAAAACATAGTTCCTGGCTGGCAATGCAAGCTGGCCAAGAACTGTCAAGAGATTCCTTTCTACCATGTATGG 2880 $844 \mathrm{~N} L \mathrm{R}$ L E I O N I V P G W Q C K L A K N C O E I P F Y H V W 873 2881 AGCGGTACGTCCAACACGTTGCACTGTTCATTTGCCTTGGTCAAGACGGAGCCGGGTATCGGACCCATAGGCTGCAGGTTAGAGGTGACC 2970 $\begin{array}{llllllllllllllllllllllllllllllll}874 & S & G & T & S & N & T & L & H & C & S & F & A & L & V & K & T & E & P & G & I & G & P & I & G & C & R & L & E & V & T & 903\end{array}$ 2971 CAAGgCACGTATACGACCAGTATGCAGGTCATGGAGAGGATCATTGATGGGGTGGTTGTGGAGCAGGAGTTGAGACTGAACGGCTACAGC 3060 $\begin{array}{llllllllllllllllllllllllllllllll}904 & Q & G & T & Y & T & T & S & M & Q & V & M & E & R & I & I & D & G & V & V & V & E & Q & E & L & R & L & N & G & Y & S & 933\end{array}$ 3061 CCTAACCACATGGTACCGCCTCAGAATGCCTTCCACCTGTCCAAACCAGTCAGAACCGCCTTGTGCCAATGCCTTGATATCCCCCAGCCC 3150 $\begin{array}{llllllllllllllllllllllllllllllll}934 & P & N & H & M & V & P & P & Q & N & A & F & H & L & S & K & P & V & R & T & A & L & C & Q & C & L & D & I & P & Q & P & 963\end{array}$ 3151 AAGgGTAATGACTGGAAACTACTCGCAACCAAACTAGGTGTCGATGGATATCTCAACTTCTTTGGCATCAAACCAAGTCCAACCGATCAA 3240

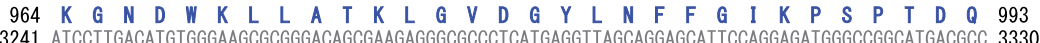
2241 ATCCTTGACATGTGGGAAGCGCGGGACAGCGAAGAGGGCGCCCTCATGAGGTTACAGGAGCACCAGAGT 994 I L D N W E A R D S E E G A L M R L A G A F 3331 GTCACGATAGTCCAGAAGCAGATGGCTGCATGGATCTGA 3369 1024 V

Fig. 1. DNA and protein sequences of Hp-Unc-5v1 inferred from 3,369 bp of cDNA, including a 5' UTR of 261 bp and an ORF of 3,105 bp, encoding 1,035 amino acids. Orange letters, signaling peptide region; Pink, immunoglobulin-like domain-1 and -2; Green, thrombospondin type-1 domain-1 and domain-2; Light blue, transmembrane domain; Dark orange, ZU-5 domain; Red, DCC-binding domain; Blue, Death domain.

\section{Structure of Hp-unc-5}

The DNA and protein structure analyses of Unc-5 homolog in this study identified two variants: $\mathrm{v} 1$ and $\mathrm{v} 2$. The canonical Hp-Unc-5v1 (AB751505) was found to consist of a 5'UTR of $261 \mathrm{bp}$ and an ORF of 3,105 bp that encoded 1,035 amino acids (Fig. 1). On the basis of iPSORT analysis (http://ipsort.hgc.jp/), 30 amino acids at the $\mathrm{N}$-terminus $\left(\mathrm{M}^{1}-\mathrm{G}^{30}\right)$ were found to constitute the signal peptide, which was followed by immunoglobulin-like-1 (Ig-like-1; $\left.Q^{57} Y^{150}\right)$ and lg-like-2 $\left(P^{165}-Y^{244}\right)$, thrombospondin-1 (TSP-1; $\left.W^{252}-Q^{303}\right)$ and TSP-2 domains ( $\left.V^{308}-\left.\right|^{361}\right)$, a transmembrane domain ( $\mathrm{Y}^{389}-\mathrm{V}^{411}$; http://www.cbs. dtu.dk/services/TMHMM/), a ZU-5 domain ( $\left.D^{627}-P^{730}\right)$, and a Death domain $\left(A^{943}-K^{1029}\right)$ near the $\mathrm{C}$-terminus (Fig. 1). According to Protein Blast analysis, the entire amino acid sequence of $\mathrm{Hp}-U n c-5 \mathrm{v} 1$ was highly similar to Unc5C-like of S. purpuratus (XP_003729771; $E=0.0$ ) and Saccoglossus kowalevskii(NP_001161598; E=0.0), Unc-5 homolog c of Caenorhabditis elegans (XP_975281; $\left.\mathrm{E}=2 \mathrm{e}^{-86}\right)$, Unc5C-like of Nile tilapia, Oreochromis niloticus (XP_003446151; E=6e $\mathrm{e}^{-83}$ ), Unc5B precursor of Xenopus laevis (NP_001082302; $E=1 \mathrm{e}^{-82}$ ), Unc5C precursor of chicken (NP_989782; $E=1 \mathrm{e}^{-80}$ ), Unc5C precursor of mouse Mus musculus (NP_03349; $\mathrm{E}=1 \mathrm{e}^{-80}$ ), and Unc5C of human (AAC67491; $E=2 e^{-78}$ ).

Since a DCC-binding domain (DB domain) that mediates attraction to netrin (Chan et al. 1996) was predicted to be located between the $\mathrm{ZU}-5$ and Death domains in mouse Unc-5 (AJ487852; Hong et al., 1999), further sequence analysis using ClustalX multiple alignment was applied and identified a region that exhibited a structure highly similar to the DB domain ( $T^{798}-E^{816}$; Fig. 1 ) in the region between ZU-5 ( $\left.D^{627}-\mathrm{P}^{730}\right)$ and Death domains $\left(A^{943}-K^{1029}\right)$ of $\mathrm{Hp}-U n c-5 v 1$. From the presence of the DB domain, the occurrence of $\mathrm{Hp}-\mathrm{Unc}-5 \mathrm{v} 1$ interaction with DCC can be predicted, as annotated by SpBase (SPU_025975; http://www.spbase.org/SpBase/search/ viewAnnoGenelnfo.php?spu_id=SPU_025975).

According to phylogenetic analysis using full-length amino acid sequences of Unc-5, Unc-5A, B, C, and D and $\mathrm{Da}$ homologs of vertebrates and invertebrates, as well as DCC of mice and human as outgroups, Hp-Unc$5 \mathrm{v} 1$ was localized in the Unc-5 group of basal deuterostomes, which include sea urchin and the hemichordate S. kowalevskii (Supplementary Fig. S1).

In the present study, a shorter sequence of $\mathrm{Hp}-u n c-$ 
1 GAAAATTCATTGGTAGCATTGGAGCTAGATGCCTCACGCATGCTGGGATTGGCTGTTATCCAATACCACATTCTCATCTGCATGGAATCT 90 91 TAACAAGTTCAGGGACTTCGCATCTAATATCTCTTTTTCAAGGCAGTCTTGATGGAGACCCGAGTGGCTTAGGAGCGAGTCAGGAGCCGC 180 181 CGAAAAGTCATCGTGATTATTACTTGGTGGAGCTACTACCCCGAAAGGATACTGGAGATCTATACCCACATCACCGGCAAGATGAGACGC 270 1 M R R 3

271 CGAAGTGCCGGGCTAAGGATTCATGAGGTTACCGTTGTGCTTGTGCTGTTTGGTGCGATTGCATCGAGGCCAGTTTTAGGACAACTTGAT 360

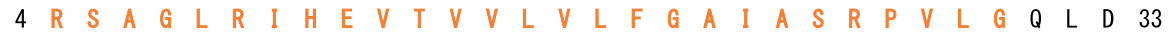
361 GGACAACCACCACAAGATGTCCCAACAACCATGACACCTCTTCTTTCGCCGCAATTCTTCATCGTTCCGCAAGATTCGTACATCACAAAA 450 $\begin{array}{lllllllllllllllllllllllllllllllll}34 & G & Q & P & P & Q & D & V & P & T & T & M & T & P & L & L & S & P & Q & F & F & I & V & P & Q & D & S & Y & I & T & K & 63\end{array}$ 451 AGAAGGGTGGATCTAGAATGCAAGGCCGGTCCTTCTCCCAATGTGTACTTTATATGCAATGATGAGAAGATAGCTGATGCCAGGACACAT 540 $\begin{array}{llllllllllllllllllllllllllllllll}64 & R & S & V & D & L & E & C & K & A & G & P & S & P & N & V & Y & F & I & C & N & D & E & K & I & A & D & A & R & T & H & 93\end{array}$ 541 TCTCGTGGTACCTTTGATGAATACTATGAGAATATCAGACATATAGCTTTAACCATAACAAAAGATGAGGTGCAAGAGTACTTTGGTGAT 630 $\begin{array}{llllllllllllllllllllllllllllllll}94 & S & R & G & T & F & D & E & Y & Y & E & N & I & R & H & I & A & L & T & I & T & K & D & E & V & Q & E & Y & F & G & D & 123\end{array}$ 631 GAGGATTTCTGGTGCGTCTGCGAAGCCGCTGCTGATCCACAGCCAATCAGGACAGAGAAGGCCTATATCAGAGAAGCATACCTGCGTAAA 720 $\begin{array}{llllllllllllllllllllllllllllllll}124 & E & D & F & W & G & V & C & E & A & A & A & D & P & Q & P & I & R & T & E & K & A & Y & I & R & E & A & Y & L & R & K & 153\end{array}$ 721 CAGTTCCTCCAGATGCCACTGGACCACAGTGTGCCCCTCCATGACAAGTTCCATCTCCTGTGCCGGGCACCAGAGGGCGTCCCAGAGCCG 810 $\begin{array}{llllllllllllllllllllllllllllllll}154 & Q & F & L & Q & M & P & L & D & H & S & V & P & L & H & D & K & F & H & L & L & C & R & A & P & E & G & V & P & E & P & 183\end{array}$ 811 ACCATCCATTGGGAGATTGACGGAGTGCCCATTGACGACGAGAACCTCGTGCACTACGTGGTAACATACGACGGGACCCTCATCGTCAAT 900 184 T I H W 901 GAAGCTACACTGGCAGATAATGGCAACTATACATGTGTCGCCACCAATGTTGCCACTTACAGAACAACAGATCCAGCTCGTGTCATTGTA 990 $\begin{array}{llllllllllllllllllllllllllllllll}214 & E & A & T & L & A & D & N & G & N & Y & T & C & V & A & T & N & V & A & T & Y & R & T & T & D & P & A & R & V & I & V & 243\end{array}$ 991 TACGATAATACAAACGATGGAGCATGGACGATGTGGACAGAGTGGAGCACCTGTACCGGAGACTGTGACGGTGGGACCAGGAGAaGGATG 1080 $\begin{array}{llllllllllllllllllllllllllllllll}244 & Y & D & N & T & N & D & G & A & W & T & M & W & T & E & W & S & T & C & T & G & D & C & D & G & G & T & R & R & R & M & 273\end{array}$ 1081 CGATACTGCACCAACCCTGCCCCTCTATCGATGGAGCCAACTGCGCGGGAAAGGCATTGCAGACCGAAGACTGTTCAATCGATTGCCCAG 1170 274 R $Y$ Y C C T N 1171 GTGCGGCCATTGGTTGGAGCGAATGGAGCGTGTGGTCCCGCATGCACGGACGAGTGTGAACAGATCAGGACCAGGACCTGCACCAGGCAA 1260 $\begin{array}{llllllllllllllllllllllllllllllll}304 & V & R & P & L & V & G & A & N & G & A & C & G & P & A & C & T & D & E & C & E & Q & I & R & T & R & T & C & T & R & Q & 333\end{array}$ 1261 TGTTCGGGCGCATCGCAGCAACGGAGGGACTGCTCTGGAGGACTCTGTTTAAGTGAGCCACCACGTATGGTGTCTCCCTCTATACTTCTT 1350 $\begin{array}{llllllllllllllllllllllllllllllll}334 & C & S & G & A & S & Q & Q & R & R & D & C & S & G & G & L & C & L & S & E & P & P & R & M & V & S & P & S & I & L & L & 363\end{array}$ 1351 TTGTTTGAAGGCCATGTACAGTAATTGCGAGTATTGCAGCGCTGGGAAAATACTGTTTGTCTATACTCAATATGCTAAAAAATCTATTCT 1440 364 L $F$ F

1441 GAAATTCACTAAAAAGGATCATTACTCAAAAAAAAAAAAAAAAAAAAA

Fig. 2. DNA and protein sequences of $H p$-unc-5v2 inferred from 1,488 bp of cDNA, including a 5 UTR of $261 \mathrm{bp}$, a 3' UTR of $117 \mathrm{bp}$, and an ORF of 1,110 bp, encoding 370 amino acids. Orange letters, signal peptide region; Purple, immunoglobulin-like domain-1 and-2; Green, thrombospondin type-1 domain-1. Underlined sequence, subjected to potential splice-isoform analysis.

5-like construct (Hp-unc-5v2; AB751506) was also cloned, which consisted of a 5' UTR of $261 \mathrm{bp}$, a 3' UTR of $117 \mathrm{bp}$, and an ORF of 1,110 bp that encoded 370 amino acids (Fig. 2). Its N-terminus end constituted a signaling sequence ( $\mathrm{M}^{1}-\mathrm{G}^{30}$; http://psort.ims.utokyo.ac.jp) that was initiated by four hydrophilic amino acids, three positively charged arginines $\left(\mathrm{R}^{2-4}\right)$, and a hydrophilic serine $\left(\mathrm{S}^{5}\right)$, which was identical to that of $\mathrm{Hp}-U n c-5 \mathrm{v} 1$, and then two immunoglobulin-like domains $\left(\lg -1, Q^{57}-Y^{150} ; \lg -2, P^{165}-Y^{244}\right)$ and one TSP-1 domain $\left(W^{252}-Q^{303}\right)$, which were also identical. However, no cytoplasmic domains were detected at the $\mathrm{C}$-terminus region, in which the ORF terminated before the transmembrane domain of Hp-unc-5v1. Further homology search using sequences at the 3' ORF and 3' UTR regions of Hp-unc-5v2 and the genome sequence of Sp-unc-5, which consisted of 17 exons (Supplementary Fig. S2), detected a highly homologous sequence in an intron region located adjacent to exon 6 of Sp-unc-5 (XM_003729723; Supplementary Fig. S3). The Hp-unc-5v2 sequence terminated with poly-A, which suggested that the two $\mathrm{Hp}$-unc-5 variants may be a post-transcriptionally modified pair of splice-isoforms (Fig. 3; Blobel and Dobberstein, 1975).

\section{Temporalexpression pattern of Hp-unc-5}

To verify the transcriptional activity of Hp-unc-5v1 and -v2 throughout the developmental period, reverse transcriptionpolymerase chain reaction (RT-PCR) was applied to the mRNA of samples collected at stages from unfertilized eggs to 3-day post-fertilization (-dpf) plutei. Hp-unc-5v1 was transcribed actively in unfertilized eggs and fertilized eggs, decreased considerably during the mesenchyme blastula stage, and its expression was restored weakly during the early gastrula stage. The transcriptional activity increased considerably from the late gastrula stage and remained high until the 3-dpf pluteus stage (Fig. 4A, v1). However, Hp-unc-5v2 was not transcribed in unfertilized eggs and only weakly in fertilized eggs; then, like the level of Hp-unc-5v1, it decreased considerably during the mesenchyme blastula stage. The accumulation of amplicons increased from the early gastrula stage to the prism stage, followed by a significantly higher level at the 3-dpf pluteus stage (Fig. 4A, v2). Thus, there was a mid-blastula transition regarding the trends of $H p-u n c-5 v 1$ and $v 2$ transcript levels during the mesenchyme blastula stage. The amplicon accumulation was easily detected by 30-cycle amplification using a set of primers for Hp-unc-5v1, whereas those for $\mathrm{Hp}-u n c-5 v 2$ required two more amplification cycles to be detectable. Although the exact transcription activity ought to have been examined by quantitative PCR, if, for example, the level of transcription of Hp-unc-5v2 were less than $25 \%$ of that of $\mathrm{Hp}$-unc-5v1, this difference would be multiplied a billionfold after 30 cycles of amplification, which would provide results suggesting significantly lower transcriptional

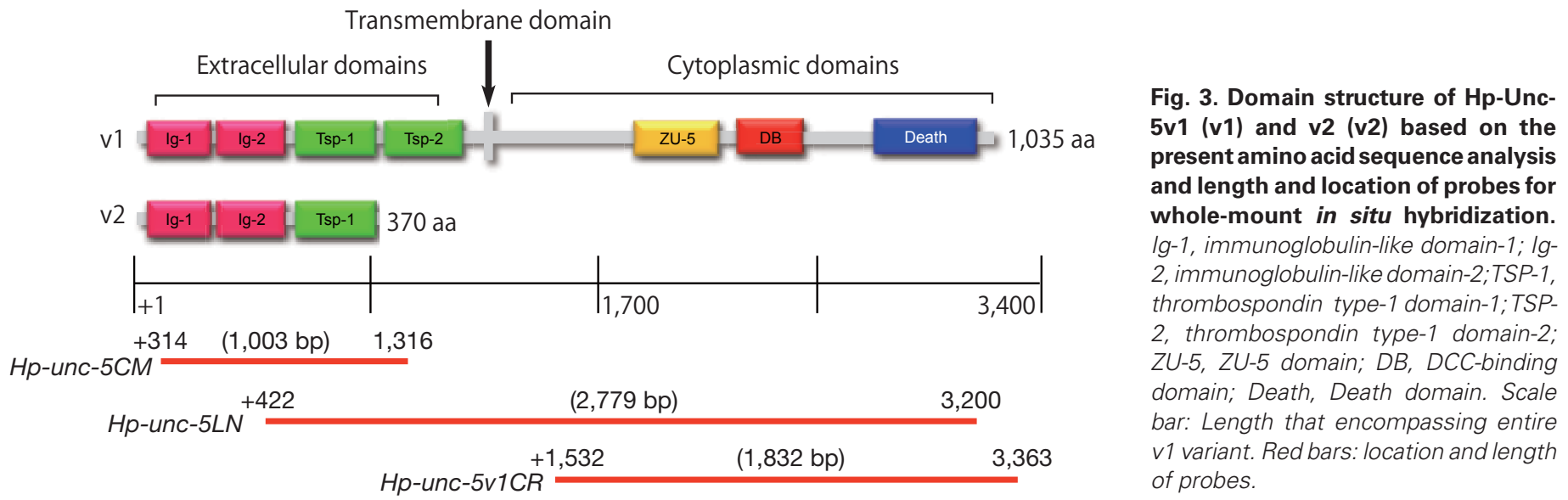


activity and thus lower translational activity. This may have meant that the degree to which the intensity of Hp-unc-5v2 transcription was lower than that of $\mathrm{Hp}-u n c-5 v 1$, as presented in Fig. 4A, may be exaggerated, as shown in the following analysis.

Protein expression was examined using antisera raised against $\mathrm{Hp}-U n c-5 v 1$ and Hp-Unc-5v2 in this study. In immunoblotting, anti-Hp-Unc-5v1 antiserum recognized two bands at regions that corresponded to $83 \mathrm{kDa}$ and $30 \mathrm{kDa}$ (Fig. 4B, lane 1). Thus, translation of Hp-Unc-5v2 was detected. Accordingly, the intensity of the immunoreaction of $\mathrm{Hp}-U n \mathrm{n}-5 \mathrm{v} 1$ was significantly stronger than that of $\mathrm{Hp}-U n c-5 v 2$. The relative molecular mass $(\mathrm{Mr})$ of $\mathrm{Hp}-U n c-$ $5 \mathrm{v} 1$ without a signal peptide region was a little smaller than the theoretical value of $108 \mathrm{kDa}(\mathrm{PI}=5.65)$ calculated using the ExPASy Compute pl/Mw tool (http://web.expasy.org/compute_pi/), while that of $\mathrm{Hp}-U n c-5 v 2$ without a signal peptide region was close to the theoretical value of $38 \mathrm{kDa}(\mathrm{Pl}=5.03)$. Although anti-Hp-Unc-5v2 antiserum recognized only a single band corresponding to $30 \mathrm{kDa}$ (Fig. 4B, lane 2), the intensity of immunoreaction of Hp-Unc-5v2 by anti-Hp-Unc-5v1 antiserum was significantly weaker than that for Hp-Unc-5v1 (Fig. 4C), whereas anti-Hp-Unc-5v2 antiserum indicated higher intensity (Fig. 4D). The expression of both proteins was weaker in unfertilized eggs and fertilized eggs (Figs. 4C, D). However, both Hp-Unc-5v1 (Fig. 4C) and Hp-Unc-5v2 (Fig. 4D) exhibited significantly increased immunoreactions at and after the swimming blastula stage. According to blot image analysis using ImageJ 1.43u open access software (National Institutes of Health, USA; http://rsb.info.nih.gov/ij), the relative intensity of $\mathrm{Hp}$ Unc-5v1 and v2 immunoreaction against tropomyosin indicated low translation activity until the stage of fertilized eggs, which then radically increased at the swimming blastula stage and remained similarly high until the 3-dpf pluteus stage (Fig. 4E). Regarding a few hours difference between the swimming blastula stage and the mesenchyme blastula stage, apparent discrepancy between decreased transcription activity and strong protein expression in mesenchyme blastulae could be due to longer protein life span that was translated during the swimming blastula stage (Fig. 4C).

\section{In situ hybridization}

The spatiotemporal mRNA transcription pattern of Hp-unc-5v1 was analyzed by in situ hybridization with a probe of $2,779 \mathrm{bp}$, which encompasses almost the entire ORF of Hp-Unc-5v1 (Fig. 3). Consistent with the results from the RT-PCR analysis as described above, a weak positive signal was detected in ooplasm (Fig. 5A). No accumulation of transcripts was detected from around the 10hpf morula stage (Fig. 5B) through the 13-hpf swimming blastula stage (Fig. 5C) to the 15-hpf mesenchyme blastula stage (Fig. 5D). At the 18-hpf early gastrula stage, however, weak accumulation of the transcripts was seen at the vegetal plate and the archenteron in the early stage of invagination (Fig. 5E). In 24-hpf late gastrulae, accumulation of the transcript intensified around the blastopore and was lost in the mid- to foregut region (Fig. 5F). At the stage of 28-hpf prism larvae, accumulation of transcripts shifted toward the mid- and foregut region, and, in contrast to that in the previous stage, it was lost from the blastopore region (Fig. $5 \mathrm{G}, \mathrm{H}$ ). Transcript accumulation was also seen at the dorsal aboral ectoderm region (Fig. $5 \mathrm{H}$, arrow). According to the vegetal plate view, transcript accumulation was not detected in the vegetal plate ectoderm (Fig. 5I). At the 2-dpf 2-arm pluteus stage, while accumulation of the transcripts was retained at the gut region, it was recognized in an additional area, namely, the basal side of the oral lobe ectoderm, with more intensive accumulation at the right and left corners of the oral lobe (Fig. 5J). In 3-dpf 4-arm plutei, transcript accumulation was seen along the circumoral ciliary band region, while that at the digestive organs decreased (Fig. 5K). Sense probes did not detect the transcript accumulation throughout these developmental stages (Fig. $5 \mathrm{a}-\mathrm{i})$. Thus, $\mathrm{Hp}$-unc-5v1 transcription sites were not limited
Fig. 4. Transcription and translation patterns of Hp-Unc-5 variants. (A) Amplicon accumulation pattern by reverse transcription-polymerase chain reaction (RT-PCR) during development. V1 30-cycle, Hp-unc-5v1 amplicons with 30-cycle RT-PCR; V2 32-cycle, Hp-unc-5v2 amplicons with 32-cycle RT-PCR; RTase-, without reverse transcriptase; Hpubi, with Hp-ubiquitin primers as a positive control. (B) Immunoblotting patterns of Hp-Unc-5v1 (lane 1) and Hp-Unc-5v2 (lane 2) using anti-Hp-Unc-5v1 antiserum. V1: $V 1$ variant at region corresponding to approximately $83 \mathrm{kDa}$. V2: V2 variant at region corresponding to approximately $30 \mathrm{kDa}$. (C) Expression pattern of V1 variant (V1) and V2 variant (V2) during developmental stages. As a positive control, tropomyosin (TM) was probed with anti-sea urchin tropomyosin antiserum. Immunospecificity test by nonpre-adsorbed antiserum (Npad) and by pre-adsorbed antiserum (Pad: negative control). (D) Hp-Unc-5v2 expression pattern during developmental stages by anti-Hp-Unc-5v2 antiserum. (E) Relative intensity of immunoreaction calculated between Hp-Unc-5v1 (V1/TM) and v2 (V2/TM) during development. Ordinate: Ratio of relative intensity of total Hp-Unc5 and ratio of Hp-Unc-5v1 and v2 variants. Vertical bars: s.d. uf, unfertilized eggs; f, fertilized eggs; $m B I$, mesenchyme blastulae; eG, early gastrulae; $s B I$, swimming blastulae; Prsm, prism larvae; 2d, 2-day post-fertilization (-dpf) plutei; 3d, 3-dpf plutei.

$$
\begin{aligned}
& \text { A } \\
& v 1 \\
& v 2 \\
& \text { C }
\end{aligned}
$$
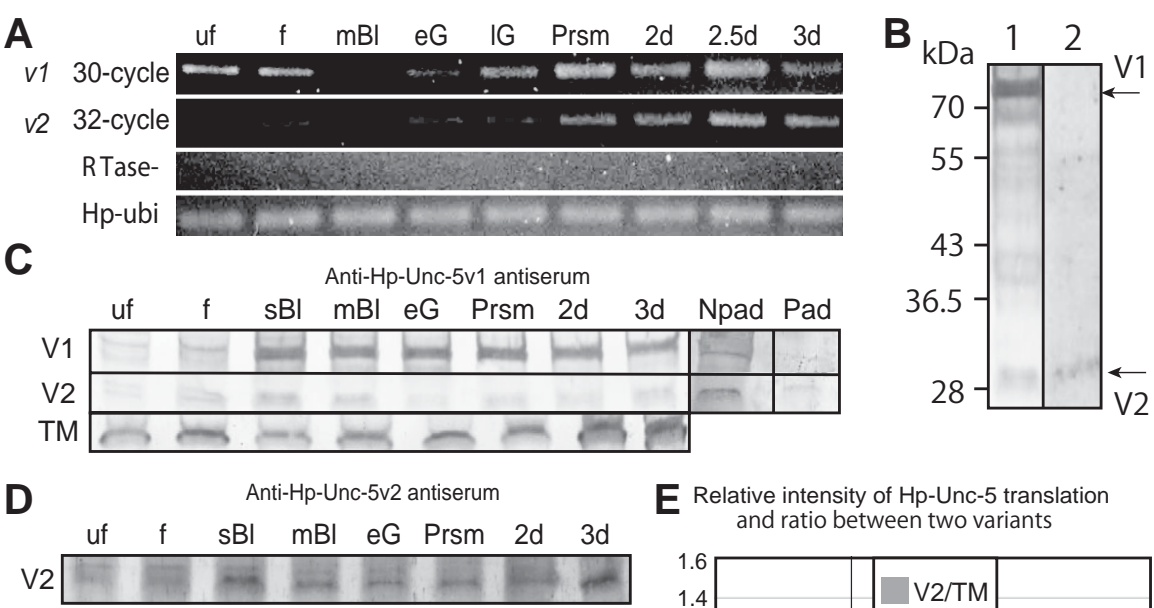

E Relative intensity of Hp-Unc-5 translation and ratio between two variants

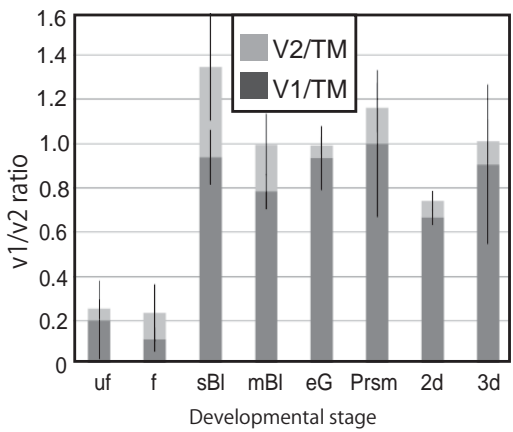




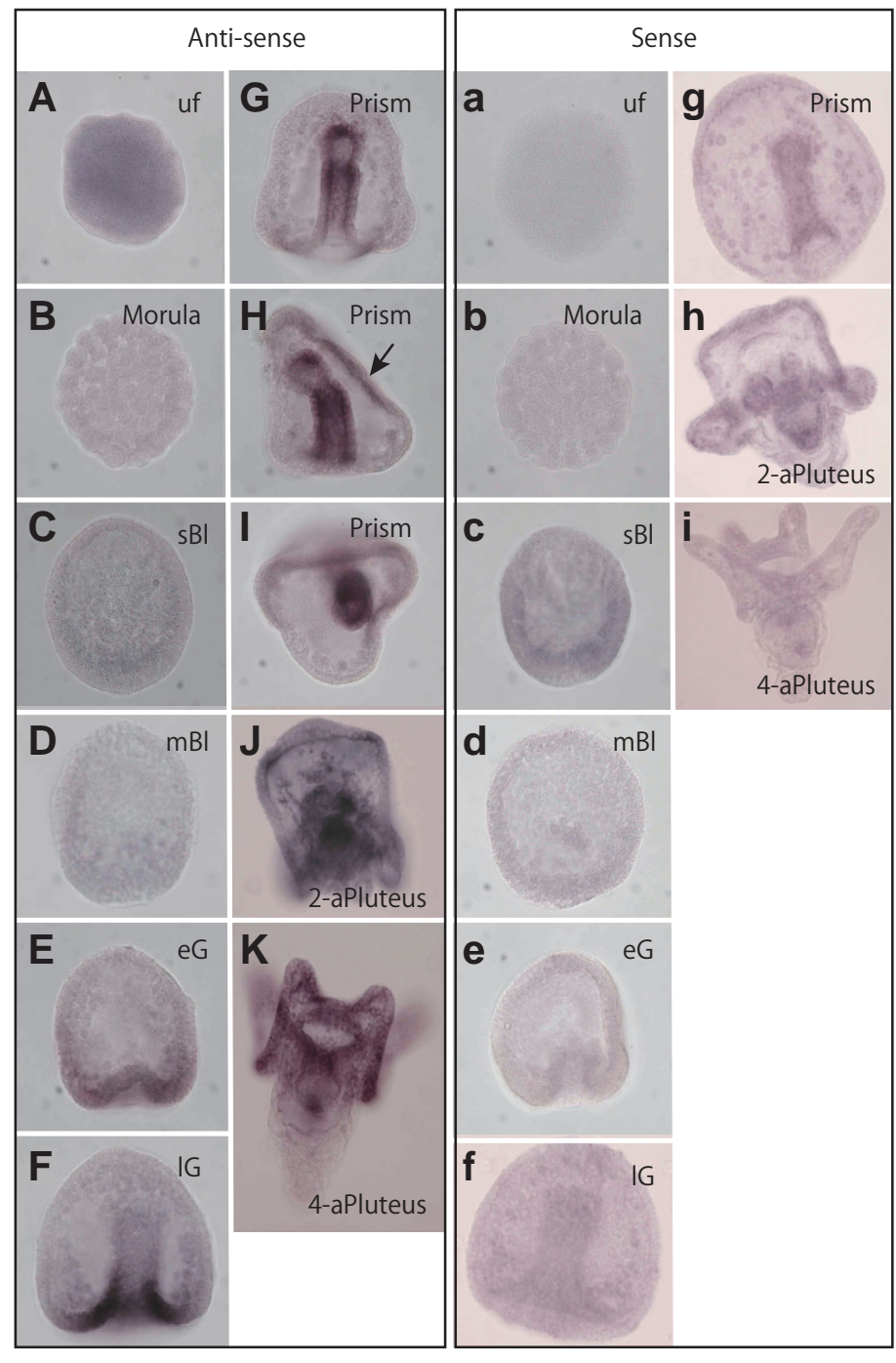

Fig. 5. Whole-mount in situ hybridization of Hp-unc-5v1. (A,a) Unfertilized eggs (uf). (B,b) 10-hour post-fertilization (-hpf) morulae. (C,c) 13-hpf swimming blastulae (sBI). (D,d) 15-hpf mesenchyme blastulae (mBI). (E,e) 18-hpf early gastrulae (eG). (F,f) 24-hpf late gastrulae (IG). (G,g) Oral side view of 28-hpf prism larvae. (H) Left side view of 28-hpf prism larva. (I) Vegetal pole view of 28-hpf prism larva. (J,h) 2-day post-fertilization (-dpf) 2-arm pluteus (2-aPluteus). (K,i) 3-dpf 4-arm pluteus (4-aPluteus). Upper case, anti-sense probes; lower case, sense probes.

to the area where serotonergic axonal projection takes place, but were also seen in the digestive organs, which was suggestive of multiple functions of the protein during development.

The in situ hybridization method applied here, using Hp-unc-5LN probe that covers both $\mathrm{v} 1$ and $\mathrm{v} 2$ sequences and Hp-unc-5v1CR probe that covers $v 1$-specific region could not specifically identify the location of the v2 variant (data not shown). To compensate for this technical limitation, we deployed overexpression of Green Fluorescent Protein (GFP)-tagged Hp-Unc-5v2 with the expectation that this would enable characterization of the expression sites and potential biological role of Hp-Unc-5v2 exclusively.

\section{Overexpression of $\mathrm{Hp}-$ Unc-5v2:GFP}

Consistent with the Hp-unc-5 transcription pattern determined by in situ hybridization as described above, Hp-Unc-5v2:GFP was detected in the cytoplasm of serotonergic apical ganglion cells on the basal side (Fig. $6 \mathrm{~A}-\mathrm{C}$, yellow arrow) as well as extracellularly on the basal surface of the oral lobe epithelium near the apical ganglion of 3-dpf plutei (Fig. 6 A-C, green arrow). In these plutei, projection of the serotonergic axons was limited to the area immediately beneath the ganglion, while control plutei projected axons away from the apical ganglion (Fig. 6D, arrows). In 4-dpf plutei, Hp-Unc-5v2:GFP was localized in axons, but not extracellularly (Fig. 6 E-G). While axons in control plutei projected far away from the ganglion and branched at this developmental stage (Fig. $6 \mathrm{H}$, arrows), those in plutei overexpressing $\mathrm{Hp}-U n \mathrm{c}-$ 5v2:GFP never projected away from the ganglion, which suggested inhibition of axonal projection by the overexpression of $\mathrm{Hp}-U n \mathrm{nc}-5 \mathrm{v} 2$ protein. The axonal expression of Hp-Unc-5v2 and its extracellular deposition were also consistently detected by whole-mount immunohistochemistry (Fig. $6 \mathrm{I}-\mathrm{K}$ ). The larvae that were not injected with GFP nor Hp-Unc-5v2:GFP did not show high green background (Fig. $6 \mathrm{~L}-\mathrm{N}$ ), suggesting positive green fluorescence emitted associated with serotonin cells (Fig. 6 A,E, I) is derived from $\mathrm{Hp}-U n \mathrm{nc}-5 \mathrm{v} 2: \mathrm{GFP}$.

This detection of short-type Hp-Unc-5v2 by overexpression of Hp-Unc-5v2:GFP and immunohistochemistry suggested that v2 has a role in axonal projection.

\section{Knockdown of Hp-Unc-5 by MASO}

To examine how canonical Hp-Unc-5v1 is involved in the axonal projection process, loss-of-function examination was conducted by knocking down translation of Hp-Unc-5v1 using MASO. In plutei injected with $0.5 \mathrm{mM}$ MASO (data not shown) and those injected with glycerol (Fig. 7A), no visible effect was seen on gangliogenesis, including axonal projection. However, upon the injection of $0.75 \mathrm{mM}$ MASO, the formation of serotonergic axons was severely inhibited (Fig. 7B). This suggests strong involvement of Hp-Unc-5v1 in axonal projection. The embryos that were injected with $1 \mathrm{mM}$ MASO died long before reaching the pluteus stage (data not shown), which indicated a lethal non-specific effect of a high dose of MASO.

\section{Interference of Hp-Unc-5/netrin interaction by an Hp-netrin peptide in serotonergic axonal projection}

During axon guidance, Unc-5 interacts with its ligand, Unc-6/ Netrin, through an eight-amino-acid sequence, SADFGKTW, in the VI domain of netrin (Lim and Wadsworth, 2002). This eight-aminoacid sequence has been detected in sea urchin netrin (Hp-netrin; Katow 2008) and thus is also implicated in the occurrence of Unc-5/netrin interaction in serotonergic axonal projections in sea urchin plutei. To examine the possible involvement of $\mathrm{Hp}-U n \mathrm{c}-5 /$ netrin interaction through this eight-amino-acid peptide in plutei, a synthetic peptide ( ${ }^{177}$ FKSQDFGKTWQPY ${ }^{190}$; active peptide) that includes a section that closely resembles this eight-amino-acid sequence was applied to plutei along with a synthetic peptide including the sequence of an eight-amino acid peptide not related to Unc-5/netrin interaction derived from the C-terminus region of Hp-netrin ( $\mathrm{L}^{601} \mathrm{KWRDEWDMRMRKF^{614 }}$; control peptide; Katow 2008). In 2.5-dpf plutei that were incubated with or without the active or control peptides for 24 hours from the prism larva stage, while serotonergic axons projected away from the ganglion in the larvae incubated without peptide (Fig. $8 \mathrm{~A}, \mathrm{a})$ and in those with 
Fig. 6. Whole-mount immunohistochemistry of Hp-Unc-5v2:GFP expression pattern (green) at serotonergic apical ganglion (red). (A-D) 3-day post-fertilization (-dpf) 2-arm pluteus. (E-H) 4-dpf 4-arm pluteus. (D), (H) Control plutei stained with anti-serotonin antibody. (I-K) 3-dpfpluteus of double-stained whole-mount immunohistochemistry with anti-Hp-Unc-5v2 antiserum (green) and serotonin (red). (L) 4-dpf pluteus that was not injected with GFP nor Hp-Unc-5v2:GFP and probed with $519 \mathrm{~nm}$ laser for negative control of green fluorescence emission. (M) Serotonin stained same larva as (L). (N) Merged-image between $(L, M)$. Green arrows in $(A, C)$, extracellular Hp-Unc-5v2. Yellow arrows in $(A, C)$, cytoplasmic Hp-Unc-5v2:GFP. Arrows in $(D, H, J)$, axons projected away from the ganglion. Bars: $30 \mu \mathrm{m}$.
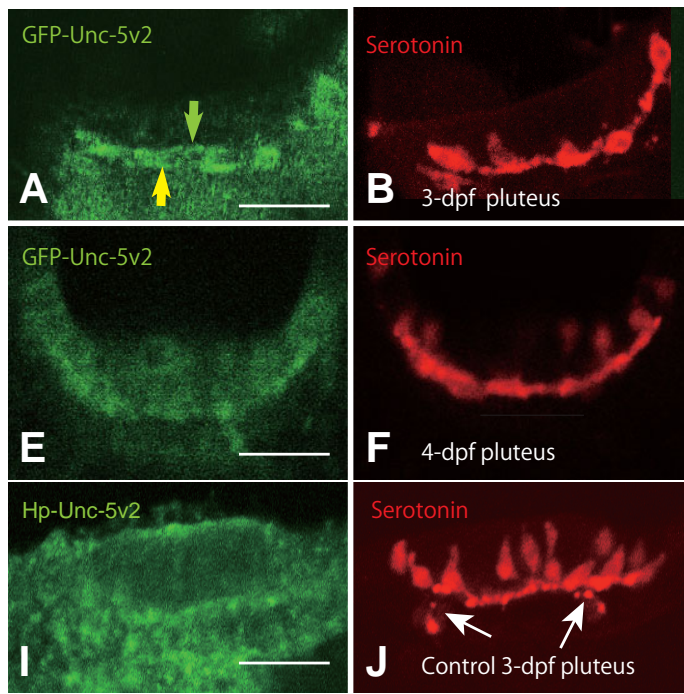

No GFP

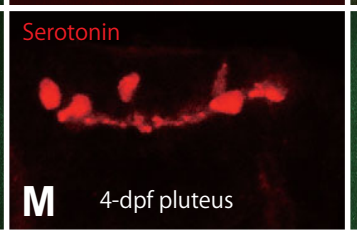

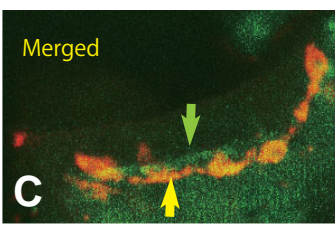
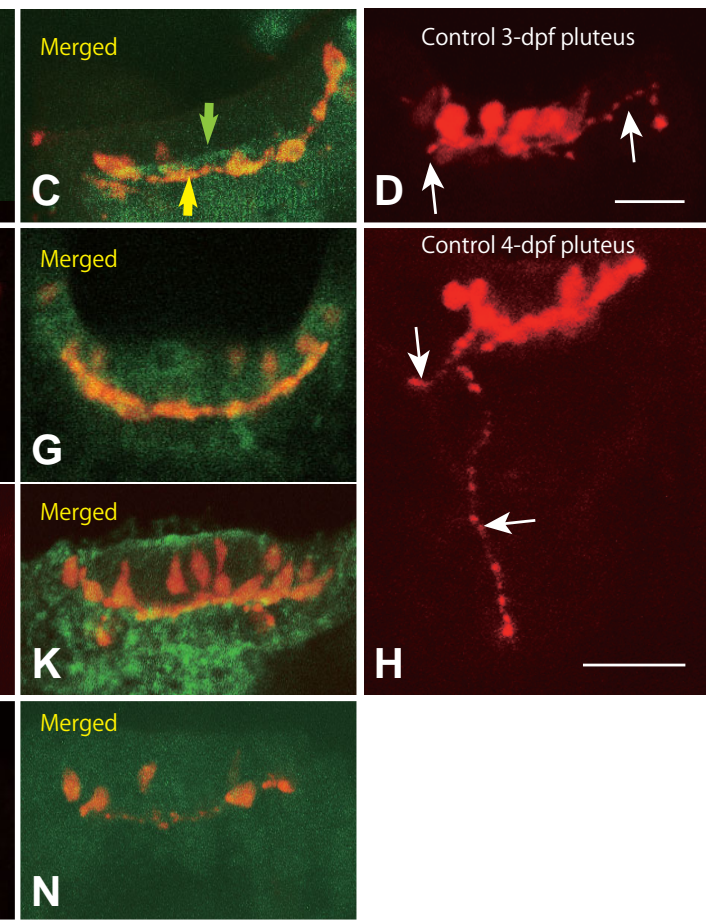

$7.5 \mu \mathrm{g} / \mathrm{ml}$ control peptide (Fig. 8B), those incubated with 2.5 to $10 \mu \mathrm{g} / \mathrm{ml}$ active peptide showed inhibition of axonal projection in a dose-dependent manner (Fig. $8 \mathrm{C}, \mathrm{C}-\mathrm{J}, \mathrm{j})$. The active peptide did not affect axonal projection at $2.5 \mu \mathrm{g} / \mathrm{ml}$ in the majority of plutei (Fig. $8 \mathrm{C}, \mathrm{c}, \mathrm{K}$ ) that was followed by the second major inhibition type of axonal projection with axons in limited area beneath serotonergic cells (type- 1 inhibition) and minor group with severe deprivation of axonal projection (type-2 inhibition; Fig. $8 \mathrm{~K}$ ). At $5 \mu \mathrm{g} / \mathrm{ml}$, normal plutei became rather rare, with those exhibiting type-1 inhibition dominating (Fig. $8 \mathrm{E}, \mathrm{e}, \mathrm{K}$ ), followed by the group with type-2 inhibition (Fig. 8F, f, K). The increase in the size of the type-1 inhibition group compared with that at $2.5 \mu \mathrm{g} / \mathrm{ml}$ was significant. However, at $7.5 \mu \mathrm{g} / \mathrm{ml}$, while normal plutei remained the smallest group, groups exhibiting type- 1 and type- 2 inhibition comprised similar proportions of the total (Fig. $8 \mathrm{G}, \mathrm{g}, \mathrm{H}, \mathrm{h}, \mathrm{K}$ ). At $10 \mu \mathrm{g} / \mathrm{ml}$, the group with type-2 inhibition was dominant, followed by normally developing plutei. Plutei showing type-1 inhibition became the smallest group in this case (Fig. 8 I,i,J,j,K). The overall response of plutei to the active peptide was dose-dependent in terms of the decrease of axonal projection or even loss of axons.

\section{Inhibitory effect of active peptide on larval swimming behavior}

The deficiency of serotonergic axonal projection due to the active peptide may reduce serotonin secretion, which perturbs the formation of the serotonin receptor cell network (SRCN) in the blastocoel (Katow et al., 2004) and thus reduces larval swimming activity (Yaguchi and Katow, 2003; Katow et al., 2007). Consistent with these previous observations, 3-dpf early 2-arm plutei treated with active peptide did not form a complete blastocoelar SRCN (Fig. 9 A,B) and were associated with a dose-dependent decrease of larval swimming activity, whereas the control peptide did not inhibit larval swimming (Fig. 9C). One-way factorial ANOVA also detected significant deference that was confirmed by Tukey test as post-hoc $[\operatorname{Pr}(>\mathrm{F})$ between control and peptide treated and between different concentration of peptide was significant at 0.001348 and 0.007698 , respectively].

\section{Discussion}

\section{Structures of Hp-Unc-5v1 and v2}

Among the several Unc-5 isoforms reported to date, those that lack the transmembrane domain and cytoplasmic domains with an amino acid sequence showing disarrayed at the $\mathrm{C}$-terminus, as in the case of the v2 variant focused on in this study, seem to be rare, but these features have been reported in human Unc-5s (Q6ZN44-1-3, UniProtKB; http://www.uniprot.org/uniprot/?query=unc-5\&sort=score).

The present immunoblotting analysis detected two isoforms with $\mathrm{Mr}$ of $20-25 \%$ less than those calculated on the basis of their amino acid sequences. This could be due to unpredicted posttranslational modification, such as phosphorylation. According to proteomics analysis using the NetPhos 2.0 Server (http://www.cbs. dtu.dk/services/NetPhos/), Hp-Unc-5v1 contains 34 serines and 17 threonines, and $\mathrm{Hp}-U n c-5 v 2$ contains 5 serines and 8 threonines. Another possibility for this apparent difference in size is that they are highly negatively charged isoforms of less than $\mathrm{PI}=6$ (Shirai et al., 2008). The PI values of Hp-Unc-5v1 and Hp-Unc-5v2 were predicted to be 5.65 and 5.03 , respectively.

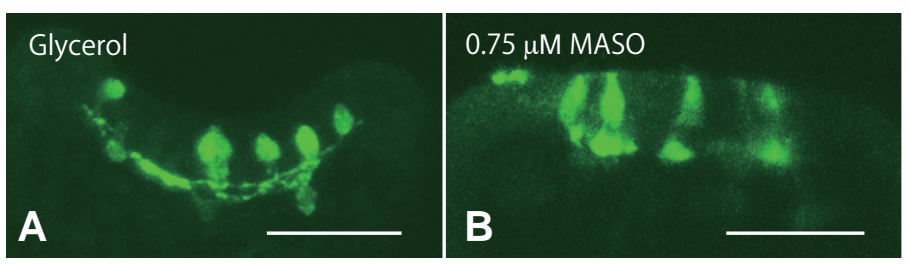

Fig. 7. Inhibition of axonal projection by Hp-unc-5 morpholino antisense oligonucleotides (MASO) in 2.5-day post-fertilization plutei. (A) Glycerol-injected larva. (B) $0.75 \mu \mathrm{M}$ MASO-injected larva. Bars: $30 \mu \mathrm{m}$. 
Both Hp-Unc-5v1 and v2 isoforms had an identical 30-amino-acid signal peptide at the $\mathrm{N}$-terminus that was comprised of a positively charged region ( $n$ region; $\mathrm{M}^{1} \mathrm{RRRS} \mathrm{S}^{5} \mathrm{Pl}=12.3$, Compute $\mathrm{pl} / \mathrm{Mw}$; http://web. expasy.org/compute_pi/), a central hydrophobic region (h-region; $A^{6}-A^{24}$ ), and a more polar C-terminal region (c-region; $S^{25}-R^{26}$ ) that contains the signal peptidase cleavage site (Von Heijne 1990; Lim and Wadsworth, 2002; Lai et al., 2012) and is considered to control protein secretion.

Unc-5 receptors require two extracellular Ig-like domains to interact with netrin (Kruger et al., 2004). In the two isoforms of Hp-Unc-5, these domains are conserved, which suggests that they both interact with netrin. Hp-Unc-5v1 is the canonical protein and thus can be anchored to the plasma membrane through its transmembrane domain. However, the absence of this domain and the presence of the signal peptide in $\mathrm{Hp}-U n \mathrm{nc}-5 \mathrm{v} 2$ suggest that this isoform is secreted into the extracellular space without being anchored to the plasma membrane. This was supported by the observation of $\mathrm{Hp}-$ Unc-5v2:GFP in the extracellular space in this study (Fig. 6 A-C). Given its absence of cytoplasmic domains, Hp-Unc-5v2 in the extracellular space may not be able to transmit netrin signals to the cytoplasm, and by pre-emptively binding to the netrin v2 variant could decrease the netrin signal via canonical v1 receptors, as discussed below.

The present transcription and translation analyses of isoforms indicated increased levels in prism larvae, the stage at which axons begin to project at the apical ganglion. Consistent with the RT-PCR analysis in this study, in situ hybridization also indicated accumulation of the transcripts at the blastopore region in early gastrulae and in archenteron and endomesoderm during the prism stage. However, these sites of accumulation of transcript were exhibited long before the onset of neurogenesis and are not related to the area where future neurogenesis takes place. This implicates nonneural activity of $\mathrm{Hp}-U n \mathrm{n}-5$ in numerous morphogenetic processes, such as cell migration, cell-cell interactions, and cell-extracellular matrix interactions (Sun et al. 2011). Neurogenesis-related Hp-Unc-5 transcripts may be those accumulated in serotonergic cell bodies and axons at the apical ganglion from the 2-dpf pluteus stage by in situhybridization and $\mathrm{Hp}-U n \mathrm{nc}-5 \mathrm{v} 2$ :GFP detection.

\section{Isoforms and their role in axonal projection}

The role of isoforms of Unc-5 has not been well understood; this includes whether they are limited to only a few species, such as human, mouse, and C. elegans, or are widespread (UniProtKB). To date, identification of the presence of two isoforms in the sea urchin is the first of its type for all marine animals.

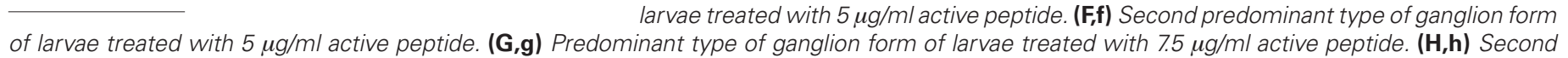

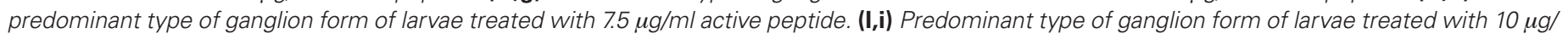

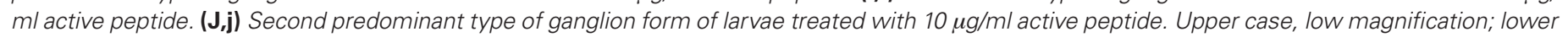
case, high magnification. Bars: $30 \mu \mathrm{m}$. (K) Proportion of ganglion types with or without active peptide. Vertical bars, s.d.
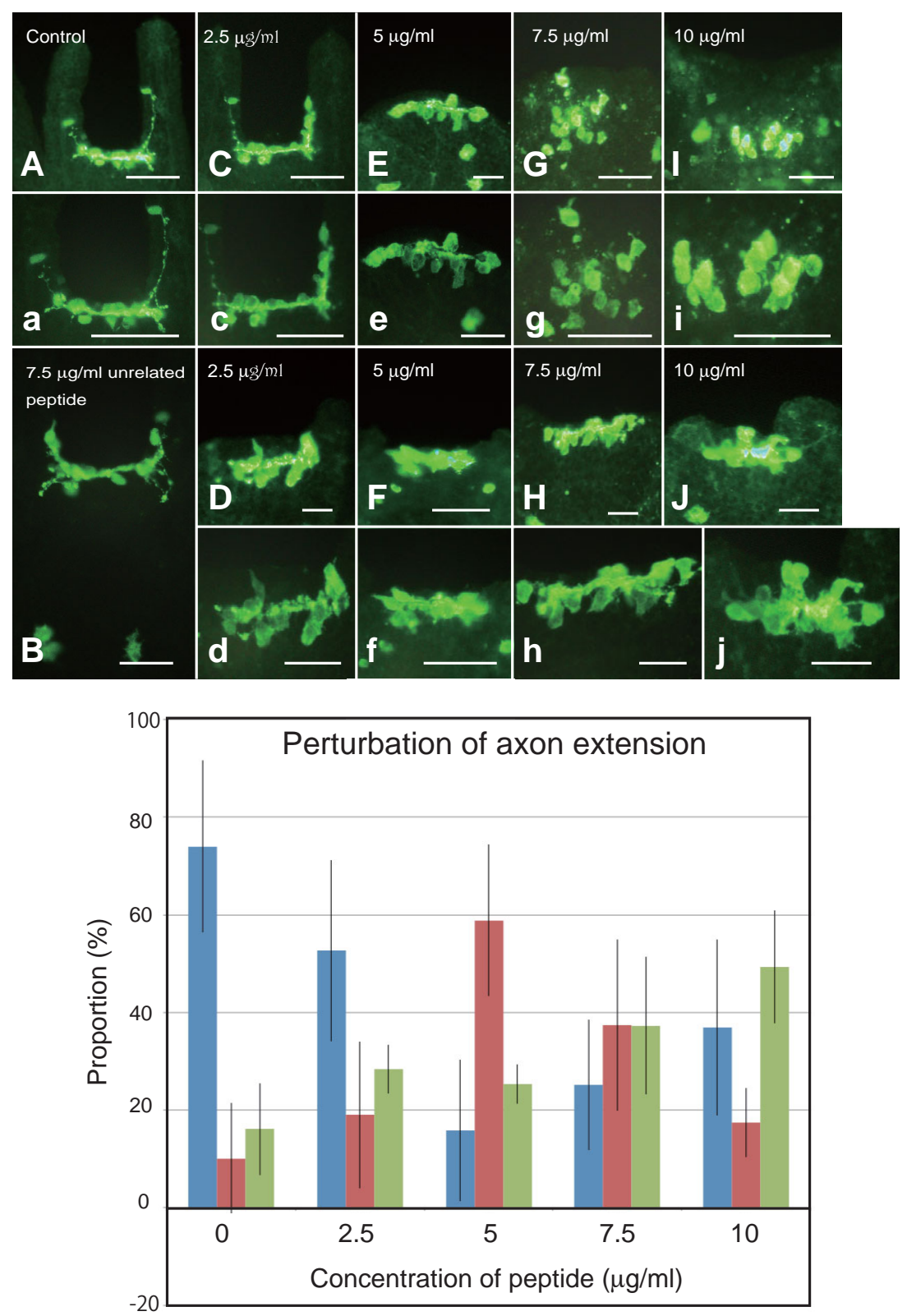

K

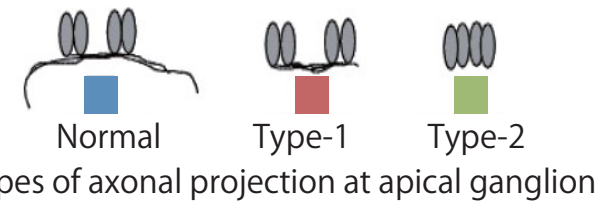

Fig. 8. Interference of Hp-Unc-5/netrin interaction with an SODFGKTW peptide of Hpnetrin (active peptide) with serotonergic axonal projection in 2.5-day post-fertilization plutei. (A-J, a-j) whole-mount immunohistochemistry of serotonin. (A,a) Control normal apical ganglion. (B) Larvae treated with peptide unrelated to Hp-Unc-5/netrin interaction (LKWRDEWDMRMRKF, control peptide). (C,c) Predominant type of apical ganglion in larvae treated with $2.5 \mathrm{um} / \mathrm{m} /$ active peptide. (D,d) Second predominant type of ganglion form of larvae treated with $2.5 \mu \mathrm{g} / \mathrm{ml}$ active peptide. (E,e) Predominant type of ganglion form of larvae treated with $2.5 \mu \mathrm{g} / \mathrm{ml}$ active peptide. (E,e) Predominant type of ganglion form of 

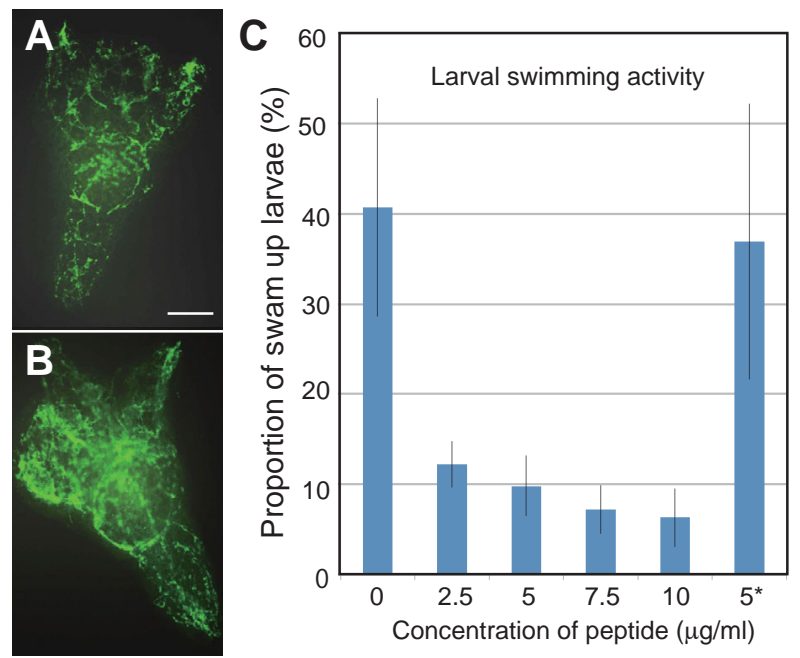

Fig. 9. Swimming activity of 2.5-day post-fertilization larvae in the presence of synthetic SODFGKTW peptide (active peptide). (A,B) whole-mount immunohistochemistry of serotonin receptor. (A) Serotonin receptor network of larvae treated with $5 \mu \mathrm{g} / \mathrm{ml}$ active peptide. (B) Control untreated larva. (C) Proportion of actively swimming larvae, which decreased with increasing concentration of peptide. $5^{*}$ shows the swimming activity of larvae treated with $5 \mu \mathrm{g} / \mathrm{ml}$ LKWRDEWDMRMRKF. Vertical bars, s.d.

In C. elegans, absence of the transmembrane domain abolishes the localization of Unc5 (Ogura and Goshima, 2006), and in the putative null allele unc-5 (e53) that has no cytoplasmic and transmembrane domains, $100 \%$ of the ventral class D and dorsal class $\mathrm{D}$ motor axons failed to reach the dorsal muscle band (Killeen et al., 2002). Thus, lack of a transmembrane domain and cytoplasmic domains in $\mathrm{Hp}-U n \mathrm{c}-5 \mathrm{v} 2$ may interfere with the function of $\mathrm{Hp}-U n c-5 \mathrm{v} 1$ to mediate netrin signaling in axonal projections by reducing netrin signaling.

Although no Unc-5 isoforms have yet been identified in Drosophila, Drosophila homolog of human Down syndrome cell adhesion molecule (DSCAM), which is involved in the formation of axon pathways in the embryonic central nervous system, has multiple isoforms with conserved immunoglobulin (lg)-like and transmembrane domains, and its alternative splicing can potentially generate more than 38,000 isoforms. Different isoforms exhibit different binding specificity. These isoforms seem to contribute to the specificity of neuronal connectivity (Schmucker et al., 2000; Wojtowicz et al., 2004).

In the present study, extracellular Hp-Unc-5v2:GFP was detected by the 3-dpf pluteus stage and disappeared from extracellular space in one day, which indicated its short-lived extracellular presence. These Hp-Unc-5v2-overexpressing plutei did not project axons away from the ganglion and thus showed similar morphological deficiencies to plutei that had been treated with a synthetic peptide derived from netrin VI domain (Fig. 8). The netrin VI domain binds to Ig-like domains of Unc-5 through the sequence contained within this synthetic peptide (Lim and Wadsworth, 2002; Geisbrecht et al., 2003). Since multiple binding sites are required to send netrin signal to Unc-5 and to the cytoplasm, the inhibition of one of these binding sites using the synthetic peptide (a pseudo-netrin) may inhibit or alter signal transmission by canonical netrin to Unc-5, such as through tyrosine phosphorylation (Kruger et al., 2004). Apparently, the pseudo-netrin peptide inhibited initial se- rotonergic axonal projection by lowering relative concentration of netrin, which suggests the involvement of Unc-5/netrin interaction during serotonergic axonal projection. The above morphological similarities between the effect of $\mathrm{Hp}-U n c-5 v 2$ overexpression and that upon application of a pseudo-netrin peptide on serotonergic axonal projection could suggest that $\mathrm{Hp}-U n \mathrm{nc}-5 \mathrm{v} 2$ modifies Unc$5 /$ netrin interaction by a molecular mechanism similar to that of pseudo-netrin peptide. Particularly regarding the repulsion of netrin by Unc-5 of axons (Barallobre et al., 2005; Norris and Lundquist, 2011), some factor(s) that changes the repulsive response to netrin to an attractive one must be involved in contralateral serotonergic axonal projection at the same time as the axons cross the netrinrich dorsal middle-belt in plutei.

Taking together the previous studies described above and the present results from $\mathrm{Hp}-$ Unc-5v2:GFP overexpression and pseudo-netrin peptide application, the contradictory responses of serotonergic axons to netrin may be dependent on the combination of $\mathrm{Hp}-U n c-5 \mathrm{v} 1$ and v2 isoforms. Apparent attraction of axons to the netrin-rich middle-belt of the larval axis may be due to the presence of the $\mathrm{Hp}-U n \mathrm{n}-5 \mathrm{v} 2$ isoform during the early period of axonal projection, as was seen in 3-dpf plutei. The isoform may reduce the repulsive response of Unc-5 to netrin at the apical ganglion during the early period of axonal projection and subsequently gradually increase this repulsive response to netrin during crossing the middle-belt leaving a netrin-rich area of the apical ganglion from which axons can be projected further to contralateral areas of larva where the netrin concentration is lower and the targets of axons reside (Katow 2008).

However, the actions of this combination of isoforms may not necessarily be the only axon guidance mechanism in sea urchin larvae. DCC of sea urchin could also coordinate with Unc-5 or play a distinctive role in altering the axon response to netrin from repulsion to attraction, or vice versa (Hong et al., 1999). However, while the presence of DCC has been predicted by SpBase (SPU_025975; Katow 2008), no further detail on the expression and role is available at present.

\section{Unc-5 expression sites in neuron}

The response of axons to their projection cues is considered to be localized at growth cones, which are cytoskeletal structures (Tanaka and Sabry, 1995; Norris and Lundquist, 2011), and to be carried out through tyrosine phosphorylation of cytoplasmic domains of Unc-5 (Killeen et al., 2002; Kruger et al., 2004; Barallobre et al., 2005). However, by in situ hybridization of Hp-Unc-5v1 in 2-arm plutei, overexpression of Hp-Unc-5v2:GFP, and immunohistochemistry of Hp-Unc-5v2 in 3-dpf and 4-dpf plutei in the present study, these proteins were found to be localized throughout the serotonergic cell body or, in later developmental stages, throughout the axonal region. These observations are not only consistent with previous immunohistochemical observations of UNC5H1 (Williams et al., 2003) and UNC-5:GFP in C. elegans (Killeen et al., 2002), but also suggest that axons receive netrin information at the growth cone, as well as throughout the axonal region.

\section{Materials and Methods}

\section{Gamete preparation}

Gametes of the sea urchin H. pulcherrimus were collected and inseminated, and the zygotes were incubated at $18^{\circ} \mathrm{C}$ as described previously 
TABLE 1

\section{PRIMER SETS FOR CLONING OF HP-UNC-5}

\begin{tabular}{ll} 
Primer name: Abbreviation & Sequence \\
\hline Unc-5 forward primer: T1-1 & 5'-TCGTTCCGCAAGATTCGTACA-3' \\
Unc-5 reverse primer: T1-2 & 5'-GCTTCTCTGATATAGGCCTTC-3' \\
ZD forward primer: T1-3 & 5'-ACGAGTGTGAACAGATCAGG-3' \\
ZD reverse primer: T1-4 & 5'-TCTGTCCATTAGTTCATGTG-3' \\
ZD sequence primer: T1-5 & 5'-GCACAACCACCAGACATCAC-3' \\
DB forward primer: T1-6 & 5'-TGCTACCAGATTCAGGAGTT-3' \\
DB reverse primer: T1-7 & 5'-ATCCATCGACACCTAGTTTG-3' \\
DB sequence primer: T1-8 & 5'-CAGGTGGATAGTCATCAATC-3' \\
Exon13 forward primer: T1-9 & 5'-CCTTTCTACCATGTATGGAGC-3' \\
Exon16 reverse primer: T1-10 & 5'-ATTGGCACAAGGCGGTTCTGA-3' \\
DD reverse primer: T1-11 & 5'-TCAGATCCATGCAGCCATCT-3' \\
V2 forward primer: T1-12 & 5'-ACAGAGTGGAGCACCTGTA-3' \\
V2 specific reverse primer: T1-13 & 5'-AGCGCTGCAATACTCGCAA-3'
\end{tabular}

(Katow et al., 2010) until the developmental stages described in the text.

\section{Total RNA extraction and cDNA preparation}

Total RNA was isolated from unfertilized eggs, eggs 30 minutes after fertilization (fertilized eggs), 15-hpf mesenchyme blastulae, 18-hpf early gastrulae, 24-hpf late gastrulae, 28-hpf prism larvae, 2-dpf 2-arm plutei, 2.5-dpf 2-arm plutei, and 3-dpf 4-arm plutei by solubilization in ISOGEN (NIPPON GENE, Tokyo, Japan). cDNA was prepared by reverse transcription using Oligo d(T) primer (Novagen, Madison, WI, USA) and Superscript II (Invitrogen, Tokyo, Japan).

\section{$R T-P C R$ and DNA sequencing}

Primers used for cloning of Unc-5 are summarized in Table 1. RT-PCR was conducted using T1-1 and -2 primers that were designed on the basis of the predicted Unc-5 sequence of Strongylocentrotus purpuratus (NCBI, XM_786929), which is known to be a sister species of $H$. pulcherrimus used in this study (Katow et al., 2013), and Ex Taq DNA polymerase (TAKARA, Otsu, Japan).

The amplicons from the RT-PCR were purified using Wizard SV Gel and PCR Clean-Up System (Promega, Madison, WI, USA), and ligated to pGEM-T Easy vector (Promega). The vector was introduced into JM109 cells (Promega), transformed, and incubated in Super Optimal broth with Catabolite repression (SOC) medium (Invitrogen) for 2 hours at $37^{\circ} \mathrm{C}$. Then, $100 \mu$ l of the JM109 cell suspension was plated on Luria Broth (LB)/ ampicillin/lsopropyl $\beta$-D-1-thiogalactopyranoside (IPTG)/X-Gal plates, and incubated overnight at $37^{\circ} \mathrm{C}$. The presence of colonies of transformed cells was examined by colony direct PCR. Positive colonies were further incubated in LB medium for 16 hours at $37^{\circ} \mathrm{C}$. Plasmids were collected using GeneElute Plasmid Mini-Prep Kit (Sigma, St. Louis, USA), cyclesequenced using BigDye Terminator v3.1 Cycle Sequencing Kit (Applied Biosystems, Tokyo, Japan), and analyzed with an ABI PRISM310 DNA Genetic Analyzer (PE Applied Biosystems, Tokyo, Japan).

The ZU-5 domain of Unc-5 was sequenced as described above for cloning using T1-3 and -4 primers designed by referring to the sequences that were obtained from 3' Rapid amplification of cDNA ends (RACE) in the present study and the predicted gene sequence of Sp-Unc-5 (SPU_010776; SpBase http://www.spbase.org/SpBase/search). Supplemental sequencing was conducted using T1-5 primer. The region downstream of Unc- 5 was sequenced using T1-6 and -7 primers. Supplemental sequencing was conducted using T1-8 primer. Sequences from exon 13 to 16 were predicted in SPU_010776 and sequenced using T1-9 and -10 primers (Table 1). This set of primers was also used for RT-PCR of Hp-unc-5v1. Death domain was sequenced using $\mathrm{T} 1-9$ and -11 primers.

The expression pattern of $\mathrm{Hp}-u n c-5 \mathrm{v} 2$ throughout the different developmental stages was analyzed by RT-PCR using T1-12 primer for the upstream sequence of Hp-unc-5 and T1-13 primer for the UTR sequence immediately after the stop codon of Hp-unc-5v2.

\section{Rapid amplification of $c D N A$ ends}

The primers used for RACE are summarized in Table 2. The Oligo probe of GeneRacer Kit (Invitrogen) was exposed to the total RNA extracted from 2.5-dpf plutei, and 5'-RACE template was prepared by RT-PCR using Oligo $d(T)$ primer of GeneRacer Kit (Invitrogen). For the isolation of 5' UTR, the following PCR was conducted using T2-1 and -2 primers that were designed on the basis of the sequence of $\mathrm{Hp}-u n c-5$ that was cloned in this study. Nested PCR was conducted using T2-3 and -4 primers with the above amplicons as a template, and sequenced as described above.

An aliquot of the above cDNA prepared from the total RNA of 2.5-dpf plutei was used as a template for 3' RACE PCR with T2-5 and -6 primers, and the subsequent nested PCR was conducted using T2-5 and -7 primers. Supplemental sequencing was conducted using T2-8 primer. The amplicons were dissected from agarose gels, purified using Wizard SV Gel and PCR Clean-Up System (Promega), and sequenced as stated above.

\section{Whole-mount in situ hybridization}

Plasmids that contained (1) a 2,779-bp sequence from the 5 ' end (+422) to the 3 ' end $(+3,200)$, (2) a 1,832 -bp sequence from the 5 ' end $(+1532)$ to the $3^{\prime}$ end $(+3,363)$, and (3) a 1,003-bp sequence from the $5^{\prime}$ end $(+314)$ to the 3 ' end $(+1,316)$ were prepared (Fig. 3$)$. The Dig-tagged probes were transcribed for three hours using SP6/T7 RNA polymerase (Roche, Mannheim, Germany). The probes were named as follows: Hp-unc-5long probe $(H p-u n c-5 L N), H p-u n c-5 v 1$ probe for the cytoplasmic region $(H p-$ $u n c-5 v 1 C R$ ), and probe for the common region between $H p-u n c-5 v 1$ and Hp-unc-5v2 (Hp-unc-5CM).

Unfertilized eggs, fertilized eggs, and 10-hpf morulae were fixed overnight at $4{ }^{\circ} \mathrm{C}$ with a mixture of $4 \%$ paraformaldehyde in $32.5 \% \mathrm{FSW}, 32.5$ $\mathrm{mM}$ 3-(N-morpholino)propanesulfonic acid (MOPS; pH 7.0), and $0.5 \mathrm{M}$ $\mathrm{NaCl}$. Then, 13-hpf swimming and 15-hpf mesenchyme blastulae, 18-hpf early and 24-hpf late gastrulae, 28-hpf prism larvae, and 2-dpf 2-arm and 3-dpf 4-arm plutei were fixed with a mixture of $4 \%$ paraformaldehyde, 0.1 M MOPS ( $\mathrm{pH} 7.0$ ), and 0.5 M NaCl.

Before the onset of hybridization, the above samples were treated with $2 \mu \mathrm{g} / \mathrm{ml}$ Proteinase $\mathrm{K}$ in MOPS buffer for 15 minutes at $37^{\circ} \mathrm{C}$, washed twice with MOPS buffer, fixed with $4 \%$ paraformaldehyde in MOPS buffer for 20 minutes, washed with MOPS buffer three times, and incubated with hybridization buffer [70\% formaldehyde, 0.1 M MOPS (pH7.0), $0.5 \mathrm{M} \mathrm{NaCl}$, $0.1 \%$ Tween-20, $1 \mathrm{mg} / \mathrm{ml}$ bovine serum albumin (BSA)] for 3 hours at $51^{\circ} \mathrm{C}$ for Hp-unc-5LN and Hp-unc-5v1CR probes, and at $48^{\circ} \mathrm{C}$ for $\mathrm{Hp}$-unc-5CM probe, and hybridized with probes at $0.5 \mathrm{ng} / \mathrm{ul}$ for seven days at $51^{\circ} \mathrm{C}$ for

\section{TABLE 2}

\section{PRIMER SETS FOR RACE}

\begin{tabular}{ll} 
Primer name: Abbreviation & Sequence \\
\hline 5' GeneRacer primer: T2-1 & 5'-CGACTGGAGCACGAGGACACTGA-3' \\
5' RACE reverse primer: T2-2 & 5'-TCTCTGTCCTGATTGGCTGTGGAT-3' \\
Unc-5 nested primer: T2-3 & 5'-GAGAATGTGTCCTGGCATCAGCTA-3' \\
5' GeneRacer nested primer: T2-4 & 5'-GGACACTGACATGGACTGAAGGAGTA-3' \\
3' GeneRacer primer: T2-5 & 5'-CGCTACGTAACGGCATGACAGTG-3' \\
3' RACE forward primer: T2-6 & 5'-GCCGGTCCTTCTCCCAATGTGTACT-3' \\
3' RACE nested primer: T2-7 & 5'-GCCAATCAGGACAGAGAAGGCCTATA-3' \\
3' RACE sequence primer: T2-8 & 5'-CAGCTCGTGTCATTGTATACGAT-3'
\end{tabular}


Hp-unc-5LNand $H p-u n c-5 v 1 C R$ probes and at $48^{\circ} \mathrm{C}$ for $\mathrm{Hp}-u n c-5 \mathrm{CM}$ probe. The post-hybridization was followed by washing with hybridization buffer for six hours at $57^{\circ} \mathrm{C}$ for $\mathrm{Hp}$-unc- $5 \mathrm{LN}, 54^{\circ} \mathrm{C}$ for Hp-unc-5v1CR , and $51^{\circ} \mathrm{C}$ for Hp-unc-5CM probes. After further washing with MOPS buffer five times, the blocking was conducted twice with MOPS buffer containing $10 \mathrm{mg} / \mathrm{ml}$ BSA for 15 minutes at ambient temperature and MOPS buffer containing 10\% goat serum and $1 \mathrm{mg} / \mathrm{ml} \mathrm{BSA}$ for 15 minutes at $37^{\circ} \mathrm{C}$. Alkaline phosphatase (AP)-tagged sheep anti-Dig antibody (Roche) was diluted in MOPS buffer containing $1 \%$ goat serum and $0.1 \mathrm{mg} / \mathrm{ml} \mathrm{BSA}$, and incubated overnight at ambient temperature. Then, the samples were washed five times with MOPS buffer, replaced with fresh buffer for 30 minutes, and developed in nitroblue tetrazolium/5-bromo-4-chloro-3-indolyl phosphate dissolved in AP buffer ( $\mathrm{pH}$ 9.0) containing 10\% dimethyl formamide overnight at ambient temperature. The samples were further washed briefly with MOPS buffer twice, and replaced with $50 \%$ glycerol for examination under a light field microscope.

\section{Generation of GFP-tagged Hp-Unc-5v2}

GFP-inserted pCS2+ vector (pCS2+GFP) was generated by ligating the coding sequence of tagGFP2-C GFP vector (Evrogen, Moscow, Russia) to pCS2+ vector. The coding sequence of $H p-u n c-5 v 2$ was amplified by the following primer set from cDNA template of 36-hpf prism larvae.

F-v2: 5'-TCACCGAATTCATGAGACGCCGAAGTGCCGG-3'

R-v2: 5'-ACTCGCTCGAGCTGTACATGGCCTTCAAACA-3'

The amplicons were cleaved with EcoRI and Xhol and ligated to pCS2+GFP. The vector was cleaved with Ncol, and capped mRNA was synthesized using mMESSAGE mMACHINE SP6 Kit (Ambion, Austin, TX, USA). Then, $3 \mu \mathrm{g} / \mu \mathrm{l}$ of the synthetic mRNA was microinjected into each unfertilized eggs. They were inseminated and raised in a culture dish in an incubator at $18^{\circ} \mathrm{C}$ until the stage described in the text.

\section{Hp-unc-5 knockdown by MASO}

Hp-unc-5MASO (5'-CGGCACTTCGGCGTCTCATCTTG-3') was diluted with glycerol at $0.5 \mathrm{mM}, 0.75 \mathrm{mM}$, and $1 \mathrm{mM}$ (final concentrations in the eggs were about $10 \mu \mathrm{M}, 12 \mu \mathrm{M}$, and $15 \mu \mathrm{M}$, respectively), injected into unfertilized eggs. They were inseminated and incubated for 60 hours at $18^{\circ} \mathrm{C}$. Negative control was injected with glycerol into unfertilized eggs and incubated as described above.

At the 2.5-dpf pluteus stage, the larvae were fixed with $4 \%$ paraformaldehyde in $0.1 \mathrm{M}$ phosphate-buffered saline [PBST; $40 \mathrm{mM} \mathrm{NaH}_{2} \mathrm{PO}_{4}-2 \mathrm{H}_{2} \mathrm{O}$, $60 \mathrm{mM} \mathrm{NaHPO}-12 \mathrm{H}_{2} \mathrm{O}, 10 \mathrm{mM} \mathrm{NaCl}, 0.3 \mathrm{mM} \mathrm{KCl}, 0.05 \%$ Tween-20 $(\mathrm{pH}$ 7.0)] for 15 minutes at ambient temperature, washed three times in PBST (10 minutes each), and used for whole-mount immunohistochemistry (WMIHC) as described below.

\section{Generation of antisera}

A synthetic 24-amino-acid peptide, A ${ }^{251}$ WTMWTEWSTCTGDCDGGTRRRMR $^{274}$, which contained a sequence present in the TSP-1 domain of both v1 and v2 variants, was chosen on the basis of physico-chemical property analysis, namely, Pôle Biolnformatique Lyonnais Network Protein Sequence Analysis (http://npsa-pbil.ibcp.fr/cgi-bin/npsa_automat. pl?page=npsa_pcprof.html). For v2 variant-specific antiserum, a v2 variantspecific 13-amino-acid peptide, $S^{358}$ PSILLLFEGHVQ ${ }^{370}$, from the carboxyl terminus was used (Supplementary Fig. S4). These synthetic peptides were tagged with keyhole limpet hemocyanin through the addition of cysteine to $\mathrm{A}^{251}$ for anti-Hp-Unc-5 antiserum and to $\mathrm{S}^{358}$ for anti-Hp-Unc-5v2 antiserum, diluted in Gerbu adjuvant 100 (Gerbu Biotechnik GmbH, Gailberg, Germany; diluted 1:2 in sterilized distilled water), and inoculated as previously described (Katow et al., 2004).

Immunospecificity of antisera was tested by immunoblotting using $1 \mathrm{mg} /$ ml lyophilized mesenchyme blastulae, as described previously (Katow 2008). Anti-sera were diluted in Tris buffer [TBST; $50 \mathrm{mM}$ Tris- $\mathrm{HCl}$ (pH 7.0), $0.17 \mathrm{M}$ $\mathrm{NaCl}$, and $0.05 \%$ Tween 20] at 1:3000 for anti-Hp-Unc-5v1 antiserum and at $1: 100$ for anti-Hp-Unc-5v2 antiserum and incubated with $10 \mathrm{mg} / \mathrm{ml}$ antigen peptides for two hours at ambient temperature (pre-adsorbed antisera).
Immunoblotting was conducted as described below and the blots were pre-incubated with $5 \%$ skimmed milk for one hour; $200 \mu$ l of pre-adsorbed antisera or non-pre-adsorbed antisera were applied to $1 \times 1 \mathrm{~cm}$ pieces of the blots that contained corresponding area of immunopositive band detected by non-pre-adsorbed antisera and were placed in small Parafilm boats on a rocking deck for two hours at ambient temperature (Katow et al., 2013).

\section{Immunoblotting}

Unfertilized eggs, fertilized eggs, swimming and mesenchyme blastulae, early gastrulae, prism larvae, and 2-dpf and 3-dpf plutei were lyophilized, dissolved at $1 \mathrm{mg} / \mathrm{ml}$ in sodium-dodecyl sulfate acrylamide gel electrophoresis (SDS-PAGE) sample buffer with 2-mercaptoethanol, blotted to nitrocellulose filters, and incubated with antisera raised in the present study as described previously (Katow et al., 2004). The relative intensity of immunoreaction was examined using tropomyosin as a standard (Katow et al., 2013), and scanned images of the immunoblotting were analyzed using ImageJ $1.43 \mathrm{u}$ $(\mathrm{NIH})$. The analysis was repeated three times using three different immunoblots conducted using three different sets of samples.

\section{Whole-mount immunohistochemistry}

Hp-unc-5 MASO-injected 2.5-dpf plutei and synthetic peptide-treated 3 -dpf plutei were fixed with $4 \%$ paraformaldehyde and incubated with rabbit anti-serotonin-antibody (1:500 diluted in PBST; Sigma) or mouse anti-serotonin-receptor antiserum (1:500 diluted in PBST; Katow et al., 2004). These primary antibodies were visualized using Alexa Fluor 488- or 594-tagged sheep anti-mouse IgG or anti-rabbit IgG antibodies (Invitrogen; diluted 1:500 in PBST), and examined under a Micro Radiance 2000 Confocal Laser Scanning Microscope (BioRad, Hercules, CA, USA) as described previously (Katow et al., 2013). The resulting images were analyzed using an ImageJ $1.43 \mathrm{u}(\mathrm{NIH})$ and Photoshop CS5 Extended (Adobe Systems Inc., San Jose, CA, USA).

\section{Inhibition of Unc-5 interaction by synthetic peptide during serotonergic axonal projection and of larval swimming}

To examine possible interaction between $\mathrm{Hp}$-netrin and $\mathrm{Hp}-U n c-5$, a synthetic peptide ( $\left.\right|^{178} \mathrm{FKSQDFGKTWQPY}{ }^{191}$; active peptide) containing a sequence located at the $\mathrm{VI}$ domain near the amino terminus of $\mathrm{Hp}$-netrin (Katow 2008) was applied to the larvae obtained from three different females in 24-well plates for 28 hours at $18^{\circ} \mathrm{C}$ from the 34-hpf prism stage to the 2.5-dpf pluteus stage. This was done in the presence of $2.5 \mu \mathrm{g} / \mathrm{ml}, 5 \mu \mathrm{g} / \mathrm{ml}$, $7.5 \mu \mathrm{g} / \mathrm{ml}$, or $10 \mu \mathrm{g} / \mathrm{ml}$ synthetic peptide and the $\mathrm{L}^{600} \mathrm{KWRDEWDMRMRKF}^{614}$ peptide, which contains a sequence located at the carboxyl terminus of $\mathrm{Hp}$-netrin that is not related to Unc-5/netrin interaction (inactive peptide; Katow 2008). For swimming assay, triplicate wells of 24-well plates were filled with $3 \mathrm{ml}$ of larvae, and those that swam up near the water surface at the time described above were counted under a dissection microscope. The experiment was repeated three times using a total of 545 larvae for $0 \mu \mathrm{g} / \mathrm{ml}, 652$ for $2.5 \mu \mathrm{g} / \mathrm{ml}, 534$ for $5 \mu \mathrm{g} / \mathrm{ml}, 954$ for $7.5 \mu \mathrm{g} / \mathrm{ml}$, and 739 for $10 \mu \mathrm{g} / \mathrm{ml}$ peptide.

Immediately after counting their number, the larvae were processed for WMIHC as described above. Statistical analysis was conducted by ANOVA that was calculated using $\mathrm{R}$ ver 2.13.2. (http://aoki2.si.gunma-u. ac.jp/R/?src?tukey.R).

\section{Acknowledgements}

We thank Dr. A. Yamazaki (Tohoku University) and Dr. S. Yaguchi (University of Tsukuba) for microinjection of Hp-unc-5v2:GFP and Hp-unc-5 MASO, respectively. We also thank N. Tominaga and M. Washio (Tohoku University) for ANOVA analysis of larval swimming and collecting the sea urchins, respectively.

\section{References}

ARAÚJO SJ, TEAR G. (2003). Axon guidance mechanisms and molecules: Lessons from invertebrates. Nat Rev Neurosci 4: 910-922. 
BARALLOBRE MJ, PASCUAL M, DEL-RIO JA, SORIANO E. (2005). The Netrin family of guidance factors: emphasis on Netrin-1 signaling. Brain Res Rev 49: 22-47.

BLOBELG, DOBBERSTEINB. (1975). Transfer of proteins across membranes. I. Presence of proteolytically processed and unprocessed nascent immunoglobulin light chains on membrane-bound ribosomes of murine myeloma. J CellBio/67:835-851.

CHANSS-Y, ZHENG H, SU M-W, WILKR, KILLEEN MT, HEDGECOCK EM, CULOTTI JG. (1996). UNC-40, a C. elegans homolog of DCC (Deleted in Colorectal Cancer), is required in motile cells responding to UNC-6 Netrin cues. Cell 87: 187-195.

GEISBRECHT BV, DOWD KA, BARFIELD RW, LONGO PA, LEAHY DJ. (2003). Netrin binds discrete subdomains of DCC and UNC5 and mediates interactions between DCC and Heparin. J Biol Chem 35: 32561-32568.

HONG K, HINCK L, NISHIYAMA M, POO M-M, TESSIER-LAVIGNE M, STEIN E. (1999). A ligand-gated association between cytoplasmic domains of UNC5 and DCC family receptors converts Netrin-induced growth cone attraction to repulsion. Cell 97: 927-941.

KATOW H, YAGUCHI S, KIYOMOTO M, WASHIO M. (2004). The 5-HT receptor cell is a new member of secondary mesenchyme cell descendants and forms a major blastocoelar network in sea urchin larvae. Mech Dev 121: 325-337.

KATOW H, YAGUCHI S, KYOZUKA K. (2007). Serotonin stimulates [Ca $\left.{ }^{2+}\right]_{i}$ elevation in ciliary ectodermal cells of echinoplutei through a serotonin receptor cell network in the blastocoel. J Exp Biol 210: 403-412

KATOW H. (2008). Spatio-temporal expression of a Netrin homolog in the sea urchin Hemicentrotus pulcherrimus (HpNetrin) during serotonergic axon extension. Int J Dev Biol 52: 1077-1088.

KATOW H, SUYEMITSU T, OOKAS, YAGUCHI J, JIN-NAI T, KUWAHARA I, KATOW T, YAGUCHI S, ABE H. (2010). Development of a dopaminergic system in sea urchin embryo and larvae. J Exp Biol 213: 2808-2819.

KATOW H, ABE K, KATOW T, ZAMANI A, ABE H. (2013). Development of the $\gamma$ aminobutyric acid (GABA)-ergic signaling system and its role in larval swimming in sea urchin. J Exp Biol 216: 1704-1716.

KILLEEN M, TONG JF, KRIZUS A, STEVEN R, SCOTT I, PAWSON A, CULOTTI J. (2002). UNC-5 function requires phosphorylation of cytoplasmic tyrosine 482 , but its UNC-40-independent functions also require a region between the $\mathrm{ZU}-5$ and Death domains. Dev Biol 251: 348-366.

KRUGER PK, LEE J, LI W, GUAN K-L. (2004). Mapping Netrin receptor binding reveals domain of Unc5 regulating its tyrosine phosphorylation. J Neurosci 24: 10826-10834
LAI J-S, CHENG C-W, SUNG T-Y, HSU W-L. (2012). Computational comparative study of tuberculosis proteomes using a model learned from signal peptide structures. PlosOne 7: e35018.

LIM Y-S, WADSWORTH WG. (2002). Identification of domains of netrin UNC-6 that mediate attractive and repulsive guidance and responses from cells and growth cones. J Neurosci 22: 7080-7087.

NORRIS AD, LUNDQUIST EA. (2011). UNC-6/netrin and its receptors UNC-5 and UNC-40/DCC modulate growth cone protrusion in vivo in C. elegans. Development 138: 4433-4442.

OGURAK, GOSHIMAY. (2006). The autophagy-related kinase UNC-51 and its binding partner UNC-14 regulate the subcellular localization of the Netrin receptor UNC-5 in Caenorhabditis elegans. Development 133: 3441-3450.

SCHMUCKER D, CLEMENS JC, SHU H, WORDY CA, XIAO J, MUDAM, DIXON JE, ZIPURSKYSL. (2000). Drosophila Dscam is an axon guidance receptor exhibiting extraordinary molecular diversity. Cell 101: 671-684.

SHIRAIA, MATSUYAMA A, YASHIRODAY, HASHIMOTOA, KAWAMURAY, ARAIR KOMATSUY, HORINOUCHIS, YOSHIDA M. (2008). Global analysis of gel mobility of proteins and its use in target identification. J Biol Chem 283: 10745-10752.

SUN KLW, CORREIA JP, KENNEDY TE. (2011). Netrins: versatile extracellular cues with diverse functions. Development 138: 2153-2169.

TANAKA E, SABRY J. (1995). Making the connection: Cytoskeletal rearrangements during growth cone guidance. Cell 83: 171-176.

VAN VACTOR D, LORENZ LJ. (1999). Introduction: invertebrate axons find their way. Cell Mol Life Sci 55: 1355-1357.

VON HEIJINE G. (1990). The signal peptide. J Membrane Biol 115: 195-201.

WILLIAMS ME, WU SC-Y, McKENNA WL, HINCK L. (2003). Surface expression of the netrin receptor $\mathrm{UNC} 5 \mathrm{H} 1$ is regulated through a protein kinase $\mathrm{C}$-interacting protein/protein kinase-dependent mechanism. J Neurosci 23: $11279-11288$.

WOJTOWICZ WM, FLANAGAN JJ, MILLARD SS, ZIPURSKY LS, CLEMENS JC. (2004). Alternative splicing of Drosophila Dscam generates axon guidance receptors that exhibit isoform-specific homophilic binding. Cell 118: 619-633.

YAGUCHI S, KANOH K, AMEMIYA S, KATOW H. (2000). Initial analysis of immunochemical cell surface properties, location and formation of the serotonergic apical ganglion in sea urchin embryos. Dev Growth Differ 42: 479-488.

YAGUCHI S, KATOW H. (2003). Expression of tryptophan 5-hydroxylase gene during sea urchin neurogenesis and role of serotonergic nervous system in larval behavior. J Com Neurol 466: 219-229. 


\section{Further Related Reading, published previously in the Int. J. Dev. Biol.}

\section{Nervous vascular parallels: axon guidance and beyond}

Marco Arese, Guido Serini and Federico Bussolino

Int. J. Dev. Biol. (2011) 55: 439-445

Early expression of axon guidance molecules in the embryonic chick mesencephalon and pretectum Kerry-Lyn Riley, Sarah Gledhill and Frank R. Schubert

Int. J. Dev. Biol. (2010) 54: 743-753

Axon guidance in the inner ear

Donna M. Fekete and Andrea M. Campero

Int. J. Dev. Biol. (2007) 51: 549-556

New views on retinal axon development: a navigation guide

Fanny Mann, William A. Harris and Christine E. Holt

Int. J. Dev. Biol. (2004) 48: 957-964

Axon guidance receptors direct growth cone pathfinding: rivalry at the leading edge Helen M Cooper

Int. J. Dev. Biol. (2002) 46: 621-631

Development of axon pathways in the zebrafish central nervous system Jensen Hjorth and Brian Key

Int. J. Dev. Biol. (2002) 46: 609-619

Orienting axon growth: spinal nerve segmentation and surround-repulsion D Tannahill, J M Britto, M M Vermeren, K Ohta, G M Cook and R J Keynes

Int. J. Dev. Biol. (2000) 44: 119-127

5 yr ISI Impact Factor $(2011)=2.959$

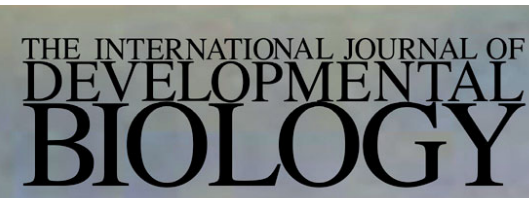

Volume 47 Nos. $7 / 8$ Special Issue

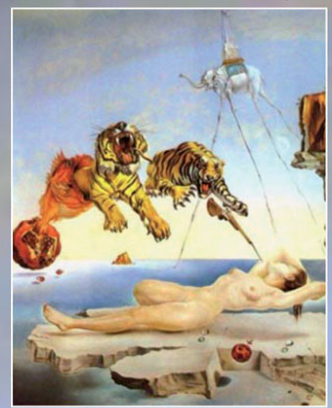

Evolution \& Development

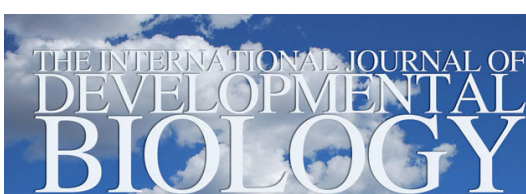

Volume 56 Nos. 10/11/12 Special Issue

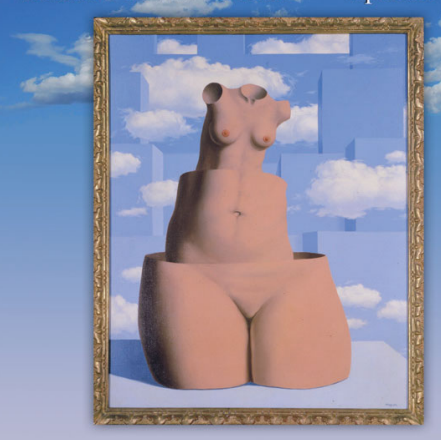

Female Germ Cells in Development \& Tumors
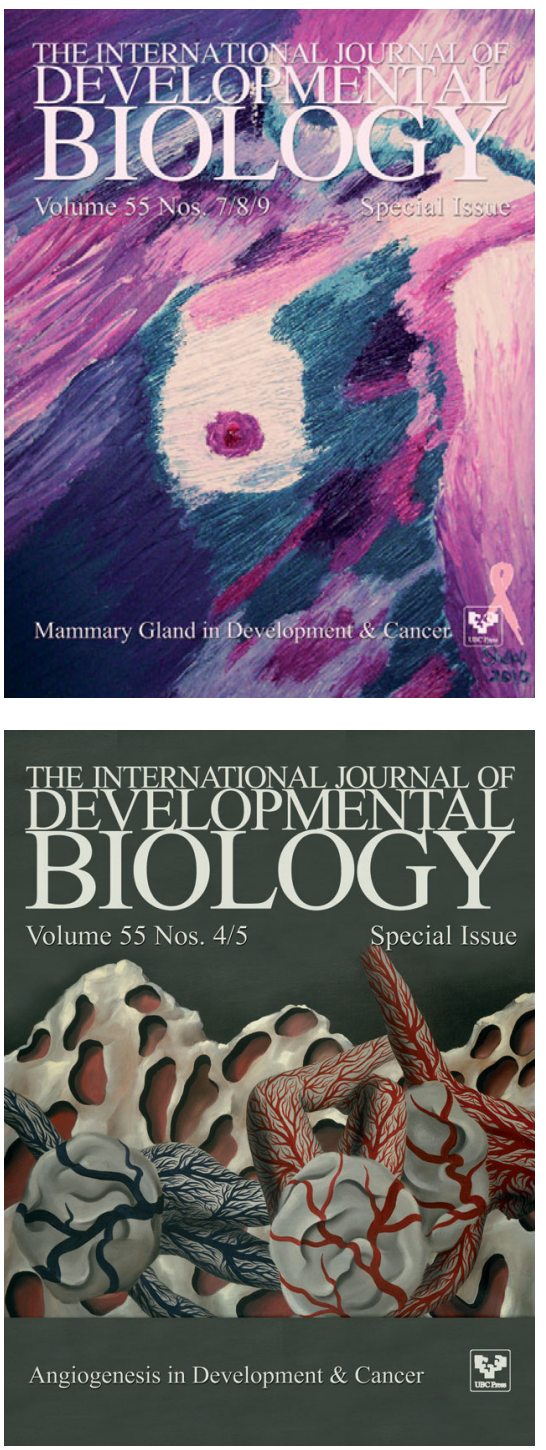